Preprint typeset in JHEP style - HYPER VERSION

hep-th/0109055

\title{
Gauged Supergravity and Singular Calabi-Yau Manifolds
}

\author{
Thomas Mohaupt* and Marco Zagermann ${ }^{\dagger}$ \\ Fachbereich Physik, Martin-Luther-Universität Halle-Wittenberg, \\ Friedemann-Bach-Platz 6, D-06099 Halle, Germany \\ E-mail: mohaupt@physik.uni-halle.de, zagermann@physik.uni-halle.de
}

\begin{abstract}
Compactifications of M-theory on singular manifolds contain additional charged massless states descending from M-branes wrapped on vanishing cycles. We construct the first explicit example of a complete supergravity Lagrangian that includes such extra states. This is done for a compactification on a Calabi-Yau threefold that develops a genus zero curve of $A_{1}$ singularities at the boundary of the Kähler cone with a resulting $S U(2)$ gauge symmetry enhancement. The corresponding $S U(2)$ gauged supergravity Lagrangian includes two charged and two neutral vector multiplets, and turns out to be uniquely fixed by the CalabiYau geometry and by the effective ungauged Lagrangian describing the Coulomb branch. One can see explicitly how resolving the singularity corresponds to a supersymmetric Higgs effect in the gauged supergravity description. The elementary transformation relating the two families of smooth Calabi-Yau resolutions of the singularity acts as the $S U(2)$ Weyl twist. The resulting structure appears to be very rigid and is likely to apply to other types of singularities and manifolds as well.
\end{abstract}

KeYwords: M-Theory, Gauge Symmetry, Supergravity Models, Supersymmetric Effective Theories.

*New address after October 21, 2001: Theoretisch-Physikalisches Institut, Friedrich-Schiller-Universität Jena, Max-Wien-Platz 1, D-07743 Jena, Germany.

†Work supported by the "Schwerpunktprogramm 1096" of the German Science Foundation (DFG) 


\section{Contents}

1. Introduction 1

2. Ungauged five-dimensional supergravity and smooth Calabi-Yau threefolds

2.1 Maxwell-Einstein supergravity

2.2 Calabi-Yau compactification

2.3 Our models

3. Gauged five-dimensional supergravity and singular Calabi-Yau threefolds 9

3.1 The gauging in general

10

3.2 The gauging in our model

11

3.3 Truncation versus integrating out

3.4 The embedding 16

3.5 The Higgs effect 17

4. Elementary transformations, reflected cones and the Weyl twist

4.1 General discussion of elementary transformations 20

4.2 Application to our models and consequences for space-time geometries 22

4.3 The Weyl twist 24

5. Conclusions and outlook 25

A. The embedding 28

B. All the allowed $\kappa$ lead to equivalent $\hat{\mathcal{M}} 33$

\section{Introduction}

One of the most remarkable properties of string theory is that it can make sense of certain singular space-time backgrounds. The most prominent examples in the context of Calabi-Yau compactifications are flop transitions [1], conifold singularities [2, 3] and their generalizations [4, 5]. If the singularity of the Calabi-Yau space can be resolved in more than one way, transitions between spaces of different topology are possible. Many examples of this have been discussed not only for type II string theory, but also for M-theory and F-theory compactifications [6, 7, 8]. The basic mechanism that leads to regular physics in an apparently singular space-time involves the presence of branes which can wrap around cycles. In the limit where 
such a cycle becomes singular, the brane contributes additional light degrees of freedom to the low energy spectrum. An effective supergravity action which only contains those modes that are massless for generic moduli becomes singular in this region of moduli space, whereas the effective action including the additional light modes is expected to be regular.

The intuitive picture behind this is that, in order to really observe the singular behaviour of such a background geometry, a physical observer would have to probe the singularity with test particles (or test strings or test branes). Such observable singularities would for example be a feature of ordinary supergravity compactifications on singular Calabi-Yau spaces. But once brane degrees of freedom in the full string or M-theory are taken into account, any test particle used to probe the would-be singularity instead interacts with the brane wrapped around the vanishing cycle, with the result that all physical observables are regular.

Apart from their importance for a deeper understanding of geometry in a theory of quantum gravity, singular Calabi-Yau spaces and topological transitions have also been used to study non-perturbative aspects of supersymmetric field theories [9, 8]. Moreover, branes wrapping vanishing cycles have appeared in recent proposals for solving the gauge hierarchy problem [10] (using a compact version of the Klebanov-Strassler solution [11]), the problem of the cosmological constant [12] and the cosmological moduli problem [13]. It is therefore desirable to have a complete understanding of the theory including the extra light modes.

If one takes a closer look at the existing literature, however, one finds that one important ingredient still seems to be missing, namely the explicit specification of a full effective Lagrangian that also encompasses the dynamics of the extra light modes. Instead, the reasoning usually goes along the lines of Strominger's classical paper on the conifold singularity [2], that is, it is

(i) shown that a singularity appearing in the effective action without the extra light modes can be interpreted as arising from illegitimately integrating out a massless supermultiplet of specific type and charge, and

(ii) it is shown that the corresponding model contains branes wrapped on vanishing cycles which give rise to precisely this type of multiplet.

Using (algebraic) geometry both qualitative and quantitative understanding of the dynamics at these special loci in moduli space has been gained. But to our knowledge a full and manifestly regular effective supergravity Lagrangian capturing all relevant low energy physics at the singular point has not been written down in any of the cases. From a conceptual point of view, this is of course not very satisfactory. Moreover, in many applications that involve singular background manifolds, one always has to work with a singular Lagrangian. Although one believes to understand the nature of the singularity and its cure, such results invite criticism, and it is preferable to have a regular Lagrangian including all relevant modes as a solid starting point.

One should also be aware that a fundamental microscopic description of such singular compactifications is not available in many cases. Indeed, for M-theory compactifications one only knows about the eleven-dimensional theory that it has supergravity as its low energy limit and contains BPS solitons such as the M2-brane and the M5-brane, whereas for type 
II compactifications the conformal field theory description becomes singular for the conifold and its generalizations. This makes a macroscopic description by a supergravity Lagrangian even more valuable.

In this paper we take a first step towards constructing such Lagrangians. We will consider a specific case which can be brought under full control, namely the vector multiplet sector of five-dimensional, $\mathcal{N}=2^{1}$ supergravity [14, 15, 16]. Such theories can be obtained by compactification of eleven-dimensional supergravity [17] on a Calabi-Yau threefold [18, 19], and when this threefold develops a specific type of singularity - a genus zero curve of $A_{1}$ singularities - one observes non-Abelian gauge symmetry enhancement $U(1) \rightarrow S U(2)$ without additional matter (i.e., hypermultiplets) 7]. Since the vector multiplet sector is uniquely specified in terms of a cubic polynomial (the 'prepotential') [14], the problem of finding the $S U(2)$ gauged supergravity Lagrangian that includes the two additional charged vector multiplets is tractable, and we will show that this extended Lagrangian is uniquely determined by the generic low energy effective action and the Calabi-Yau geometry.

Although other and more interesting situations such as five-dimensional supergravity coupled to both vector and hypermultiplets, Calabi-Yau compactifications with background flux or type II compactifications to four dimensions can be considerably more complicated, we obtain already plenty of interesting and encouraging results in our simplest example. Most importantly, we find that a successful reconstruction of the full $S U(2)$ gauged Lagrangian requires a careful consideration of the one-loop threshold effects described in [19, 7, 8]. Without these threshold corrections no consistent embedding into an $S U(2)$ gauged supergravity Lagrangian with the right matter content is possible. On the other hand, if one does take these corrections into account (with precisely the correct coefficient), the corresponding $S U(2)$ gauged Lagrangian turns out to be uniquely determined, i.e., no free parameter is introduced by the coupling of the two additional vector multiplets, as one might perhaps have expected naively.

As another interesting result, we will get a gauge theory picture of so-called elementary transformations of Calabi-Yau spaces in terms of the $S U(2)$ Weyl twist. At least a posteriori we realize that the problem of reconstructing the coupling to the two charged vector multiplets is dictated by $S U(2)$ gauge symmetry. A similar structure seems to be present for flop transitions, though in that case it is not related to gauge symmetry enhancement. This makes us hope that systematic rules govern the re-implementation of extra light modes.

The organization of this paper is as follows. In Section 2, we briefly summarize the relevant structures of ungauged $5 \mathrm{D}, \mathcal{N}=2$ supergravity theories and recall how they descend from (smooth) Calabi-Yau compactifications of 11D supergravity. In Section 2.3, this general discussion is then illustrated with the specific examples we consider in this paper. The Calabi-Yau picture of these models suggests that at certain points in the moduli space, at which the Calabi-Yau space becomes singular, an $S U(2)$ gauge symmetry enhancement takes place. In Section 3, the main part of this paper, we then attempt a construction of the

\footnotetext{
${ }^{1}$ By " $\mathcal{N}=2$ " we mean the minimal amount of supersymmetry in five space-time dimensions corresponding to eight real supercharges.
} 
underlying $S U(2)$ gauged supergravity Lagrangian that also includes the additional light modes responsible for the gauge symmetry enhancement. In Section 4, we extract a relation between the elementary transformations of the Calabi-Yau spaces and the $S U(2)$ Weyl twist from our model. A summary of our results and a list of possible applications and some interesting generalizations are given in Section 5. Appendices A and B contain some details on the calculations described in Section 3.

Effective Lagrangians which include additional modes that only become massless at special points in the moduli space have been discussed before in the literature (see [20] for a review). Our results extend these in various directions:

(i) We are not restricted to stringy perturbative mechanisms of gauge symmetry enhancement (though these are also covered) but can also include additional states coming from branes wrapped on vanishing cycles.

(ii) All the existing examples work either in situations where the moduli space is a symmetric space and no threshold effects are present, i.e., in $\mathcal{N} \geq 4$ compactifications [20], or

(iii) these models are explicitly considered only in such a (classical) approximation. An example for the latter are the untwisted sectors of orbifolds studied in [21.

\section{Ungauged five-dimensional supergravity and smooth Calabi-Yau three- folds}

The coupling of an arbitrary number of vector and hypermultiplets to $5 \mathrm{D}, \mathcal{N}=2$ supergravity in the absence of any gauge interactions was constructed in [14, 22]. The relation to CalabiYau compactifications of 11D supergravity was explicitly worked out in 18, 19. We refer to these references for further details.

\subsection{Maxwell-Einstein supergravity}

The effective field theory of the compactification of eleven-dimensional supergravity on a smooth Calabi-Yau threefold, $X$, is described by minimal five-dimensional supergravity coupled to $n_{V}$ Abelian vector multiplets and $n_{H}$ neutral hypermultiplets. The hypermultiplets will play no rôle in the following, though we will make some remarks on generalizations involving hypermultiplets in Sections 4 and 5.

The supermultiplets we are concerned with are thus

(i) The supergravity multiplet:

$$
\left(e_{\mu}^{m}, \psi_{\mu}^{i}, A_{\mu}\right)
$$

consisting of the graviton $e_{\mu}^{m}$, two gravitini $\psi_{\mu}^{i}$ and one vector field $A_{\mu}$.

(ii) The vector multiplet:

$$
\left(A_{\mu}, \lambda^{i}, \phi\right)
$$

comprising one vector field $A_{\mu}$, two spin-1/2 fermions (gaugini) $\lambda^{i}$ and one real scalar field $\phi$. 
Here, $\mu, \nu=0, \ldots, 4$ and $m, n=0, \ldots, 4$ are, respectively, curved and flat spacetime indices, whereas $i=1,2$ is a doublet index associated with the automorphism group $U S p(2)_{R} \simeq S U(2)_{R}$ of the underlying supersymmetry algebra. All fermions obey a symplectic Majorana condition with respect to that index $i$ (see [14] for more details).

Coupling $n_{V}$ Abelian vector multiplets to supergravity then results in the total field content

$$
\left(e_{\mu}^{m}, \psi_{\mu}^{i}, A_{\mu}^{I}, \lambda^{i x}, \phi^{x}\right),
$$

where we have combined the graviphoton with the $n_{V}$ vector fields from the vector multiplets to form a single $\left(n_{V}+1\right)$-plet of vector fields $A_{\mu}^{I}$ labelled by the index $I=0,1, \ldots, n_{V}$. The index $x=1, \ldots, n_{V}$ is a curved index on the $n_{V}$-dimensional Riemannian target space, $\mathcal{M}$, of the scalar fields $\phi^{x}$. The metric on $\mathcal{M}$ will be denoted by $g_{x y}$.

Introducing the Abelian field strengths $F_{\mu \nu}^{I} \equiv 2 \partial_{[\mu} A_{\nu]}^{I}$, the bosonic part of the Lagrangian reads 14

$$
\begin{aligned}
e^{-1} \mathcal{L}_{\text {bosonic }}= & -\frac{1}{2} R-\frac{1}{4} \stackrel{\circ}{a}_{I J} F_{\mu \nu}^{I} F^{\mu \nu J}-\frac{1}{2} g_{x y}\left(\partial_{\mu} \phi^{x}\right)\left(\partial^{\mu} \phi^{y}\right) \\
& +\frac{e^{-1}}{6 \sqrt{6}} C_{I J K} \varepsilon^{\mu \nu \rho \sigma \lambda} F_{\mu \nu}^{I} F_{\rho \sigma}^{J} A_{\lambda}^{K} .
\end{aligned}
$$

The completely symmetric tensor $C_{I J K}$ in the $F F A$ term of (2.4) is independent of the scalar fields and completely determines the entire theory [14]. To be more explicit, the $C_{I J K}$ define a cubic polynomial

$$
\mathcal{V}(h):=C_{I J K} h^{I} h^{J} h^{K}
$$

in $\left(n_{V}+1\right)$ real variables $h^{I}\left(I=0, \ldots, n_{V}\right)$, which endows $\mathbb{R}^{\left(n_{V}+1\right)}$ with the metric

$$
a_{I J}(h):=-\frac{1}{3} \frac{\partial}{\partial h^{I}} \frac{\partial}{\partial h^{J}} \ln \mathcal{V}(h) .
$$

The $n_{V}$-dimensional 'very special' manifold $\mathcal{M}$ (see [23] for the origin of this name) can then be represented as the hypersurface [14]

$$
\mathcal{V}(h)=C_{I J K} h^{I} h^{J} h^{K}=1 .
$$

This hypersurface constraint can (locally) be solved for the physical scalars $\phi^{x}$. One convenient way is to take the independent ratios (special coordinates), i.e.,

$$
\phi^{x}=\frac{h^{x}}{h^{0}}
$$

in the coordinate patch $h^{0} \neq 0$, but any other coordinate choice is of course also possible.

The metric $g_{x y}$ on $\mathcal{M}$ is then simply the induced metric of (2.6),

$$
g_{x y}(\phi)=\left.\frac{3}{2} \frac{\partial h^{I}}{\partial \phi^{x}} \frac{\partial h^{J}}{\partial \phi^{y}} a_{I J}\right|_{\mathcal{V}=1}
$$


whereas $\stackrel{\circ}{a}_{I J}(\phi)$ is given by the restriction of $a_{I J}$ to $\mathcal{M}$,

$$
\stackrel{\circ}{a}_{I J}(\phi)=a_{I J} \mid \mathcal{V}=1
$$

For later reference, we define

$$
h_{x}^{I}:=-\sqrt{\frac{3}{2}} \frac{\partial h^{I}}{\partial \phi^{x}}
$$

\subsection{Calabi-Yau compactification}

When the above supergravity theories are obtained from a compactification of $11 \mathrm{D}$ supergravity on a smooth Calabi-Yau threefold $X$, then the numbers of vector and hypermultiplets are given by

$$
n_{V}=h^{1,1}(X)-1, \quad n_{H}=h^{2,1}(X)+1,
$$

where $h^{p, q}(X)$ are the Hodge numbers of $X$. The Calabi-Yau space has $h^{1,1}(X)$ real Kähler moduli and $h^{2,1}(X)$ complex moduli, which parameterize deformations of the complex structure. The vector multiplet moduli are all the Kähler moduli except the one parameterizing the overall volume. The hypermultiplet moduli space contains the overall volume of the Calabi-Yau space, the complex structure moduli, and the moduli obtained by dimensional reduction of the three-form gauge field.

In a Calabi-Yau compactification the embedding coordinates $h^{I}$ can be chosen such that they parameterize the volumes of the homological two-cycles of $X$. If $C^{I}$ is a basis of $H_{2}(X, \mathbb{Z})$, and $J$ is the Kähler form of $X$, then

$$
h^{I}=\int_{C^{I}} J=\operatorname{vol}\left(C^{I}\right)
$$

(where vol is understood to be the volume of a supersymmetric (i.e. holomorphic) curve in the homology class $C^{I}$. Such a curve has minimal volume in $C^{I}$.) In this basis it is obvious that one needs to have $h^{I}>0$ so that the volumina of all two-cycles are postive. This defines the so-called Kähler cone. At the boundary of the Kähler cone, defined by $h^{I}=0$ for some $I$, the corresponding cycle collapses and the manifold $X$ becomes singular.

The coefficients $C_{I J K}$ have the interpretation as the triple intersection numbers of $X$. Let $D_{I}$ be a basis of $H_{4}(X, \mathbb{Z})$ (the four-cycles) that is dual to the basis $C^{I}$ in the sense that

$$
C^{I} \cdot D_{J}=\delta_{J}^{I}
$$

where $\cdot$ denotes the intersection product. Then

$$
C_{I J K}=D_{I} \cdot D_{J} \cdot D_{K}=\int_{X} \omega_{I} \wedge \omega_{J} \wedge \omega_{K}
$$

where the two-forms $\omega_{I}$ are the Poincaré duals of the four-cycles $D_{I}$. They form a basis of $H^{2}(X, \mathbb{Z})$. The Poincaré duals $\omega^{I}$ of the two-cycles $C^{I}$ form a basis of $H^{4}(X, \mathbb{Z})$. 
The volumes of four-cycles $D_{I}$ are given by

$$
\operatorname{vol}\left(D_{I}\right)=\int_{D_{I}} J \wedge J=h_{I}
$$

where we expanded the Kähler form $J$ in the basis $\omega_{I}$ and introduced the dual embedding coordinates $h_{I}$ defined by

$$
h_{I}=C_{I J K} h^{J} h^{K} .
$$

The volume of the Calabi-Yau space $X$ is

$$
\operatorname{vol}(X)=\int_{X} J \wedge J \wedge J=C_{I J K} h^{I} h^{J} h^{K}
$$

Since the modulus corresponding to the total volume $\operatorname{vol}(X)$ does not sit in a vector multiplet but in a hypermultiplet, one needs to introduce rescaled fields $h^{I I}=(\operatorname{vol}(X))^{-1 / 3} h^{I}$ in order to disentangle vector multiplet moduli and hypermultiplet moduli [18]. These parameterize a hypersurface in the Kähler cone, which is just the vector multiplet moduli space:

$$
\mathcal{V}\left(h^{\prime}\right)=C_{I J K} h^{\prime I} h^{\prime J} h^{\prime K}=1 .
$$

For notational convenience and to be consistent with the notation used in Section 2.1 we will drop the primes from now on, $h^{\prime I} \rightarrow h^{I}$. It is also understood that volumes of cycles and the Kähler form $J$ have been rescaled with appropriate powers of the total volume. Thus when talking about the volume of a cycle we actually mean the volume measured in units of the overall volume of the Calabi-Yau space.

The BPS-extended $D=5, \mathcal{N}=2$ supersymmetry algebra contains a (scalar) electric central charge $Z_{(e)}$ and a (vectorial) magnetic central charge $Z_{(m)}$. To these correspond BPS bounds for the masses of electrically charged point particles and the tensions of magnetically charged strings. These central charges can be expressed as [19]

$$
Z_{(e)}=q_{I} h^{I} \text { and } Z_{(m)}=p^{I} h_{I}
$$

where $q_{I}, p^{I}$ are the electric and magnetic charge with respect to the vector fields $A_{\mu}^{I}$. In Calabi-Yau compactifications one can easily identify BPS solitons which saturate these bounds [7]. Wrapping an M2-brane on the two-cycle $C^{I}$ gives pointlike sates with charge $q_{I}= \pm 1$. In order to get a BPS state, one needs to wrap around a holomorphic curve in this class, which then has volume $h^{I}$. The mass of such a state is

$$
M=T_{(2)} \operatorname{vol}\left(C^{I}\right)=T_{(2)} h^{I} .
$$

It saturates the BPS bound $M=T_{(2)}\left|Z_{(e)}\right|$, where $T_{(2)}$ denotes the tension of the M2-brane. There are also states which carry charge with respect to several $U(1) \mathrm{s}$. These descend from branes which wrap non-trivially around several generators of $H_{2}(X, \mathbb{Z})$. In an analogous way, the M5 brane can be wrapped on four-cycles resulting in a magnetic string with charges $p^{I}$, 
where $p^{I}$ are the expansion coefficients of the cycle in the basis of $H_{4}(X, \mathbb{Z})$. The tension of such a string is $T=T_{(5)}\left|Z_{(m)}\right|$, where $T_{(5)}$ is the tension of the M5-brane.

Note that at the boundary of the Kähler cone (i.e., when $h^{I} \rightarrow 0$ ) one always gets additional charged massless states descending from wrapped M2-branes. The precise nature of these states depends on the details of the collapsing cycle. Two cases which generically appear at the boundary of the Kähler cone are well-understood [7].

(i) The homology class $C^{I}$ contains a finite number, $N$, of isolates holomorphic curves. In this case, one obtains $N$ charged hypermultiplets.

(ii) The homology class $C^{I}$ contains a continuous family of holomorphic curves with the following properties: the family is itself parameterized by a holomorphic curve of genus $g$ and therefore sweeps out a divisor $E$, which defines a class in $H_{4}(X, \mathbb{Z})$. At the boundary $h^{I}=0$ the divisor $E$ collapses into a curve of $A_{1}$ singularities. (This means that, when the singular curve is intersected with a complex surface, the local geometry around the singular point is $\mathbb{C}^{2} / \mathbb{Z}_{2}$.) In terms of homology, the collapse of the two-cycle $C^{I}$ induces the collapse of the four-cycle $E$ into a two-cycle. In this case, one obtains two charged vector multiplets, which combine with the vector multiplet containing $A_{\mu}^{I}$ to form the adjoint representation of $S U(2)$. Thus the (inverse of the) Higgs effect occurs at the boundary of the Kähler cone. In addition to the two charged vector multiplets, one also gets $2 g$ charged hypermultiplets which combine with $g$ uncharged hypermultiplets [5] to form $g$ adjoint hypermultiplets.

The additional charged massless modes can be identified by a collective mode analysis in eleven-dimensional supergravity [7].

Our aim is to construct the gauged supergravity Lagrangians that include the additional light charged multiplets. In this paper, we consider the case where only vector multiplets become massless, and where these additional vector multiplets lead to an $S U(2)$ gauge symmetry enhancement. The corresponding singularity in $X$ is thus a genus zero curve of $A_{1}$ singularities.

\subsection{Our models}

We will consider three Calabi-Yau compactifications in parallel, which all give rise to two vector multiplets and exhibit $S U(2)$ gauge symmetry enhancement at one boundary of the Kähler cone without additional hypermultiplets. The corresponding Calabi-Yau manifolds $X_{n},(n=0,1,2)$ are elliptic fibrations over the Hirzebruch surfaces $\mathbf{F}_{n}$. An explicit construction as hypersurfaces in toric varieties can be found in [24]. The prepotentials are

$$
\begin{aligned}
& \mathcal{V}_{(0)}=8\left(h^{0}\right)^{3}+6\left(h^{0}\right)^{2} h^{1}+6\left(h^{0}\right)^{2} h^{2}+6 h^{0} h^{1} h^{2} \\
& \mathcal{V}_{(1)}=8\left(h^{0}\right)^{3}+9\left(h^{0}\right)^{2} h^{1}+3 h^{0}\left(h^{1}\right)^{2}+6\left(h^{0}\right)^{2} h^{2}+6 h^{0} h^{1} h^{2} \\
& \mathcal{V}_{(2)}=8\left(h^{0}\right)^{3}+12\left(h^{0}\right)^{2} h^{1}+6\left(h^{0}\right)^{2} h^{2}+6 h^{0} h^{1} h^{2}
\end{aligned}
$$

with the corresponding Kähler cones $h^{I}>0$. 
It is convenient to introduce new embedding coordinates $S, T, U$ defined by

$$
\begin{aligned}
& 6^{1 / 3} h^{0}=U \\
& 6^{1 / 3} h^{1}=T-U \\
& 6^{1 / 3} h^{2}= \begin{cases}S-U & \text { for } n=0 \\
S-\frac{1}{2}(T+U) & \text { for } n=1 \\
S-T & \text { for } n=2 .\end{cases}
\end{aligned}
$$

Then all three prepotentials take the same form

$$
\mathcal{V}=S T U+\frac{1}{3} U^{3}
$$

while the Kähler cones are now defined by

$$
T>U>0
$$

and

$$
\begin{aligned}
S>U & \text { for } n=0 \\
S>\frac{1}{2}(T+U) & \text { for } n=1 \\
S>T & \text { for } n=2 .
\end{aligned}
$$

This choice of variables is natural in the dual heterotic description of the model. Compactification of M-theory on $X_{n}$ is dual to compactifications of the heterotic string on $K 3 \times S^{1}$ with instanton numbers $(12+n, 12-n)$ [19, 6, 07, 24].

In all three models $S U(2)$ gauge symmetry enhancement without additional matter occurs at the boundary $T=U$. From the heterotic point of view, this is the perturbative symmetry enhancement that alway occurs in a compactification on a circle of self-dual radius $R=\sqrt{\alpha^{\prime}}$. The additional massless states are momentum and winding modes of the heterotic string around the $S^{1}$.

From the five-dimensional point of view, these additional massless states appear pointlike. If one includes these extra modes, one should therefore obtain an accurate description of the physics near the boundary $T=U$ in terms of an effective 5D field theory. This field theory describes the coupling of altogether four vector multiplets to $5 \mathrm{D}, \mathcal{N}=2$ supergravity. In the following section, which constitutes the main part of this paper, we will derive this field theory.

\section{Gauged five-dimensional supergravity and singular Calabi-Yau threefolds}

From our discussion in Section 2.3 it follows that the effective field theory we are looking for is described by a coupling of four vector multiplets to $5 \mathrm{D}, \mathcal{N}=2$ supergravity. However, 
this coupling cannot be of the type described in Section 2.1, because those theories were "ungauged" (that is, no field was charged under any local gauge group). Instead, we now need a theory with a Yang-Mills-type $S U(2)$ gauge symmetry. The correct framework is therefore what is commonly referred to as "gauged" supergravity. Gauged supergravity theories can be obtained from their ungauged relatives by "gauging" appropriate subgroups of the global (i.e., rigid) symmetry groups of the latter. We briefly recall the most relevant aspects of this procedure in Section 3.1, and then, in Sections 3.2 - 3.5, specialize everything to the case we are interested in. For more details on these theories the reader is referred to refs. [15, 16, 25, 26].

\subsection{The gauging in general}

The ungauged Lagrangian (2.4) of Section 2.1 is invariant under a global symmetry group of the form

$$
S U(2)_{R} \times G .
$$

Here, $S U(2)_{R}$ denotes the R-symmetry group of the underlying Poincaré superalgebra. $S U(2)_{R}$ acts only on the index $i$ of the fermions; all other fields, including the vector fields $A_{\mu}^{I}$, are $S U(2)_{R}$ inert. The group $G$, on the other hand, is the invariance group of the cubic polynomial $\mathcal{V}(h)$ (this group might very well be trivial, depending on the polynomial). More precisely, $G$ is generated by all (infinitesimal) linear transformations

$$
\begin{aligned}
& h^{I} \longrightarrow M_{J}^{I} h^{J} \\
& A_{\mu}^{I} \longrightarrow M_{J}^{I} A_{\mu}^{J}
\end{aligned}
$$

that leave the tensor $C_{I J K}$ (and therefore the cubic polynomial $\mathcal{V}(h)$ ) invariant:

$$
M_{(I}^{I^{\prime}} C_{J K) I^{\prime}}=0
$$

Whenever such a non-trivial invariance group of $\mathcal{V}(h)$ exists, it extends to a global symmetry group of the full Lagrangian, because the latter is uniquely determined by $\mathcal{V}(h)$. In particular, all $G$ transformations act as isometries on the scalar manifold $\mathcal{M}$ (the converse is not true in general [27], though in most cases). By definition, the $\left(n_{V}+1\right)$ vector fields $A_{\mu}^{I}$ transform in a (not necessarily irreducible) $\left(n_{V}+1\right)$-dimensional representation of $G$.

If one wants to gauge a non-Abelian subgroup, $K$, of $S U(2)_{R} \times G$, some of the vector

fields $A_{\mu}^{I}$ have to transform in the adjoint representation of $K$. Since all the vector fields are $S U(2)_{R}$ inert, such a non-Abelian gauge group $K$ can only be a subgroup of $G$ with the additional constraint that the $\left(n_{V}+1\right)$-dimensional representation of $G$ contains the adjoint of $K$ as a subrepresentation. That is, one needs a decomposition of the form

$$
\left(\mathbf{n}_{\mathbf{V}}+\mathbf{1}\right)_{G}=\operatorname{adjoint}(K) \oplus \operatorname{singlets}(K) \oplus \operatorname{non-\operatorname {singlets}(K).}
$$

As indicated, the $\left(\mathbf{n}_{\mathbf{V}}+\mathbf{1}\right)$ of $G$ might in general also contain $K$-singlets as well as other nonsinglet representations of $K$ in addition to the adjoint. Such additional non-singlets cause 
technical complications for the gauging of $K$ and require the dualization of the corresponding vector fields to "self-dual" tensor fields [16]. Luckily, this case cannot occur in the examples considered in this paper, and we can from now on assume a decomposition of the form

$$
\left(\mathbf{n}_{\mathbf{V}}+\mathbf{1}\right)_{G}=\operatorname{adjoint}(K) \oplus \operatorname{singlets}(K) .
$$

We split the embedding coordinates (and also the vector fields $A_{\mu}^{I}$ ) accordingly,

$$
h^{I}=\left(h^{A}, h^{\alpha}\right), \quad A_{\mu}^{I}=\left(A_{\mu}^{A}, A_{\mu}^{\alpha}\right),
$$

where $h^{A}$ (and $A_{\mu}^{A}$ ) transform in the adjoint and $h^{\alpha}$ (and $A_{\mu}^{\alpha}$ ) are singlets of $K$.

As mentioned above, $K \subset G$ acts on the scalar manifold $\mathcal{M}$ via isometries. The corresponding Killing vectors are given by [15]

$$
K_{A}^{x}=-\sqrt{\frac{3}{2}} f_{A B}^{C} h_{C} h^{B x},
$$

where $f_{A B}^{C}$ denote the structure constants of $K$, and $h^{B x}:=g^{x y} h_{y}^{B}$ (see eq. (2.10)).

The gauging of $K$ then proceeds in two steps 15, 16]:

(i) Covariantization of all derivatives and field strengths with respect to $K$. (For the Chern-Simons term this is slightly different [15], but the details are irrelevant here.) This covariantization in general breaks supersymmetry.

(ii) Addition of further (gauge invariant) terms to the Lagrangian and to the transformation rules in order to restore supersymmetry. For the case at hand, one only needs to add the Yukawa-like term 15

$$
e^{-1} \mathcal{L}_{\text {Yuk }}=-\frac{i}{2} g \bar{\lambda}^{i x} \lambda_{i}^{y} K_{A[x} h_{y]}^{A}
$$

to the (covariantized) Lagrangian, while the (covariantized) transformation rules do not receive any further corrections. Here $g$ denotes the gauge coupling.

Once $K$ is spontaneously broken, the term (3.8) gives rise to mass terms for some gaugini, indicating the presence of a supersymmetric Higgs effect. Note that, in contrast to what happens in four dimensions [28, 29], there is no scalar potential in this kind of theories. This is consistent with the fact that a massless vector multiplet in $5 \mathrm{D}$ contains only one real scalar, which is eaten by the vector field once $K$ is broken. Thus, no mass term for scalar fields (and therefore no potential) is needed. We will take a closer look at the Higgs effect in Section 3.5.

\subsection{The gauging in our model}

After this general discussion, we now return to the particular situation we are interested in.

The models described in Section 2.3 generically have two massless, neutral vector multiplets, but at the bounary of the Kähler cone two additional charged vector multiplets become 
massless and enhance one of the $U(1)$ s to the non-Abelian gauge group $S U(2)$. In order to incorporate these two additional vector multiplets into a complete field theoretical description, one therefore needs to find a supergravity theory with $n_{V}=4$ vector multiplets in which a subgroup $K=S U(2)$ of $G$ is gauged such that a Higgs effect gives mass to two of the vector multiplets (except at those points in moduli space where the full $S U(2)$ is unbroken).

The scalar manifold of such a theory is four-dimensional and will be denoted by $\hat{\mathcal{M}}$. Likewise, we will put a hat on the corresponding prepotential $\mathcal{V}$, the $C_{I J K}$ and the $h^{I}$, i.e., we will write

$$
\hat{\mathcal{V}}=\hat{C}_{I J K} \hat{h}^{I} \hat{h}^{J} \hat{h}^{K}, \quad I, J, K=0, \ldots 4
$$

etc. in order to distinguish these quantities from the analogous quantities in the ungauged models of Section 2.3.

Our goal in this section is to determine the cubic polynomial $\hat{\mathcal{V}}(\hat{h})$, which then uniquely specifies the corresponding supergravity theory. $\hat{\mathcal{V}}(\hat{h})$ has to fulfill the following minimal requirements:

(i) $\hat{\mathcal{V}}(\hat{h})$ has to admit $K=S U(2)$ as an invariance group. (Whether or not $K$ is possibly a genuine subgroup of a bigger invariance group $G$ is not relevant for our purposes and will therefore not be investigated.) Moreover, with respect to $K=S U(2)$, the five $\hat{h}^{I}$ have to decompose into the adjoint of $S U(2)$ plus two singlets (cf. eq. (3.5)). We will take $\hat{h}^{A}(A=1,2,3)$ to form the adjoint and choose $\hat{h}^{\alpha}(\alpha=0,4)$ to be the $S U(2)$ singlets.

(ii) There is at least one point $c \in \hat{\mathcal{M}}$ where $S U(2)$ is unbroken, i.e., this point $c$ is invariant under the action of the $S U(2)$ isometry group. (This requirements actually follows from the others 26]. But for the problem we are trying to solve, it is clear that we need this to be true, so we impose it anyway because it will simplify the analysis.)

(iii) The matrices $\stackrel{\circ}{a}_{I J}$ and $g_{x y}$ should be positive definite at $c$. This is necessary to have positive definite kinetic terms at that point. From the supergravity point of view the scalar manifold is the maximal domain obtained by analytic continuation, such that $\stackrel{\circ}{I J}_{I J}$ and $g_{x y}$ remain positive definite. In other words, boundaries of the scalar manifold are loci where these matrices degenerate or become singular. Note that on the boundary of the Kähler cone $\stackrel{\circ}{a}_{I J}$ and $g_{x y}$ can be regular [30]. Therefore the scalar manifold of M-theory has a different global structure.

In ref. [26], all cubic polynomials that admit a compact gaugeable invariance group (and which give rise to positive definite $\stackrel{\circ}{a}_{I J}$ and $g_{x y}$ ) were classified. Using that classification, one immediately arrives at eq. (3.21) below as the most general solution to our constraints (i)-(iii). In order to keep our presentation self-containted we briefly explain how this result is obtained. 
Imposing $S U(2)$ invariance on $\hat{\mathcal{V}}$ implies

$$
\begin{aligned}
\hat{C}_{\alpha \beta A} & =0 \\
\hat{C}_{\alpha A B} & =C_{\alpha} \delta_{A B} \\
\hat{C}_{A B C} & =0
\end{aligned}
$$

while $\hat{C}_{\alpha \beta \gamma}$ is unconstrained. Here, $C_{\alpha}$ are some undetermined coefficients. To see this, recall that the $A$ index transforms in the adjoint, i.e., in the $\mathbf{3}$ of $S U(2)$, whereas $\alpha$ is a singlet index. While there is a unique invariant in $(\mathbf{3} \otimes \mathbf{3})_{\mathrm{sym}}$, namely the Cartan-Killing form (which can always be taken to be proportional to $\delta_{A B}$ after an appropriate change of basis) there are no invariants in the $\mathbf{3}$ and in the symmetric part of $(\mathbf{3} \otimes \mathbf{3} \otimes \mathbf{3})$.

Hence, group theory already restricts the polynomial to be of the form

$$
\hat{\mathcal{V}}(\hat{h})=\hat{C}_{\alpha \beta \gamma} \hat{h}^{\alpha} \hat{h}^{\beta} \hat{h}^{\gamma}+3 C_{\alpha} \hat{h}^{\alpha} \delta_{A B} \hat{h}^{A} \hat{h}^{B}
$$

The coefficients $\hat{C}_{\alpha \beta \gamma}$ and $C_{\alpha}$ can be fixed further by exploiting constraints (ii) and (iii). According to constraint (ii), there is at least one $S U(2)$ invariant point, $c$, on $\hat{\mathcal{M}}$. An $S U(2)$ invariant point on $\hat{\mathcal{M}}$ has to have embedding coordinates $\hat{h}^{I}(c)=\left(c^{0}, 0,0,0, c^{4}\right)$, i.e., $\hat{h}^{A}(c)=0$. The coordinates $\left(c^{0}, c^{4}\right)$ define a non-trivial direction in the $\left(\hat{h}^{0}, \hat{h}^{4}\right)$ plane, because $\hat{\mathcal{V}}\left(\hat{h}^{I}(c)\right)=1$ implies that $c^{0}$ and $c^{4}$ cannot simultaneously be zero. One can then always redefine $\hat{h}^{0}$ and $\hat{h}^{4}$ such that the new coordinate values of the $S U(2)$ invariant point $c$ are given by $\left(\hat{h}^{0}(c), \hat{h}^{4}(c)\right)=(1,0)$ (and of course still $\hat{h}^{A}(c)=0$ ).

As $c$ is on $\hat{\mathcal{M}}$, we must have

$$
\hat{\mathcal{V}}\left(\hat{h}^{I}=(1,0,0,0,0)\right)=\hat{C}_{000}=1
$$

so that in terms of the new $\hat{h}^{0}$ and $\hat{h}^{4}$ coordinates the polynomial (3.13) becomes

$$
\hat{\mathcal{V}}(\hat{h})=\left(\hat{h}^{0}\right)^{3}+a\left(\hat{h}^{0}\right)^{2} \hat{h}^{4}+b \hat{h}^{0}\left(\hat{h}^{4}\right)^{2}+c\left(\hat{h}^{4}\right)^{3}+\left(d \hat{h}^{0}+e \hat{h}^{4}\right) \delta_{A B} \hat{h}^{A} \hat{h}^{B}
$$

with some coefficients $a, \ldots, e$.

The redefinition

$$
\begin{aligned}
& \tilde{\hat{h}}^{0}=\hat{h}^{0}+\frac{a \hat{h}^{4}}{3} \\
& \tilde{\hat{h}}^{I}=\hat{h}^{I} \quad \text { for } I \neq 0
\end{aligned}
$$

then removes the term quadratic in $\hat{h}^{0}$ and changes the coefficients $(b, c, d, e)$ to some values $(\tilde{b}, \tilde{c}, \tilde{d}, \tilde{e})$. Thus, after dropping all the tildes again, the polynomial reads

$$
\hat{\mathcal{V}}(\hat{h})=\left(\hat{h}^{0}\right)^{3}+b \hat{h}^{0}\left(\hat{h}^{4}\right)^{2}+c\left(\hat{h}^{4}\right)^{3}+\left(d \hat{h}^{0}+e \hat{h}^{4}\right) \delta_{A B} \hat{h}^{A} \hat{h}^{B} .
$$

We will now make use of condition (iii). A necessary requirement for the metric $\stackrel{\circ}{a}_{I J}$ at $c$ to be positive definite is that all its diagonal elements are positive at that point. Using that 
$\hat{h}^{I}(c)=(1,0,0,0,0)$, one finds for these diagonal elements (cf. eqs. (2.6), (2.9))

$$
\begin{aligned}
& \stackrel{\circ}{a}_{00}(c)=1 \\
& \stackrel{\circ}{a}_{11}(c)=-\frac{2}{3} d=\stackrel{\circ}{a}_{22}(c)=\stackrel{\circ}{a}_{33}(c) \\
& \stackrel{\circ}{a}_{44}(c)=-\frac{2}{3} b .
\end{aligned}
$$

Hence, $d$ and $b$ have to be negative, and a suitable rescaling of $\hat{h}^{A}$ and $\hat{h}^{4}$ can be used to finally bring the polynomial (3.17) into the form

$$
\hat{\mathcal{V}}(\hat{h})=\left(\hat{h}^{0}\right)^{3}-\frac{3}{2} \hat{h}^{0}\left(\hat{h}^{4}\right)^{2}+\lambda\left(\hat{h}^{4}\right)^{3}-\frac{3}{2}\left(\hat{h}^{0}+\kappa \hat{h}^{4}\right) \delta_{A B} \hat{h}^{A} \hat{h}^{B}
$$

with some as yet arbitrary parameters $\kappa$ and $\lambda$. The polynomial is now in the so-called canonical basis [14, 26], for which $\stackrel{\circ}{a}_{I J}(c)=\delta_{I J}$, so that positivity of $\stackrel{\circ}{a}_{I J}$ and $g_{x y}$ is manifest in the vicinity of $c$.

Remark 1: Whenever $\lambda$ is negative, the redefinition $\hat{h}^{4} \rightarrow-\hat{h}^{4}$ can be used to achieve $\lambda \geq 0$, as we will assume from now on.

Remark 2: For precisely two pairs of values $(\kappa, \lambda)$, the manifold $\hat{\mathcal{M}}$ becomes a symmetric space [14, 31, 23]:

(i) $(\kappa, \lambda)=\left(\sqrt{2}, \frac{1}{\sqrt{2}}\right) \Longrightarrow \mathcal{M}=S O(1,1) \times S O(3,1) / S O(3)$.

(ii) $(\kappa, \lambda)=\left(-\frac{1}{\sqrt{2}}, \frac{1}{\sqrt{2}}\right) \Longrightarrow \mathcal{M}=S O(4,1) / S O(4)$.

Remark 3: $S U(2)$ invariant points on $\hat{\mathcal{M}}$ correspond to $\hat{h}^{A}=0$, they thus form a codimension 3 hypersurface (i.e., a line) in the four-dimensional scalar manifold $\hat{\mathcal{M}}$ given by $\hat{\mathcal{V}}\left(\hat{h}^{I}\right)=1$. On this line, the $S U(2)$ Killing vectors $K_{A}^{x}$ have to vanish, which can also be verified directly using the explicit expression (3.7) for the Killing vectors. This shows that the Yukawa-type term (3.8) vanishes there too, and the gaugini (and with them the vector fields) have to be massless at these points, confirming the naive expectation. We refer to Section 3.5 for a more detailed discussion of the Higgs mechanism.

The problem we seemingly have to solve now is the following: can one embed the theory defined by $\mathcal{V}=S T U+\frac{1}{3} U^{3}$ into the model defined by (3.21) such that the line $T=U$ on $\mathcal{M}$ coincides with the line of $S U(2)$ invariant points on $\hat{\mathcal{M}}$ ?

Since we have to allow for linear redefinitions of the embedding coordinates, this amounts to expressing $\hat{h}^{I}, I=0, \ldots, 4$ as linear combinations of $S, T, U$ such that $\hat{h}^{A}=0$ (i.e., $S U(2)$ invariance) is implied by $T=U$, and the polynomials are related by

$$
\left.\hat{\mathcal{V}}\right|_{\mathcal{M C \hat { M }}}=S T U+\frac{1}{3} U^{3}
$$

This problem gives a coupled system of cubic equations for the coefficients appearing in the linear relations between the two sets of variables. With some effort (see Appendix A) one can convince oneself that this system has no solution. Something is missing. 


\subsection{Truncation versus integrating out}

The hidden assumption in the above reasoning is that it is sufficient to set two of the five embedding coordinates to constant values in order to eliminate the dynamics of the corresponding vector multiplets. In field theory terms this means that we simply set the fields of these two vector multiplets to zero. But in a quantum theory it is not sufficient to eliminate states as external lines, since they can occur as internal lines and run in loops.

This is precisely what happens here. If one starts with a theory with four vector multiplets and Lagrangian $\hat{\mathcal{L}}$ and integrates out two vector multiplets to get an effective Lagrangian $\mathcal{L}$ for two vector multiplets, then the two Lagrangians are related by

$$
\mathcal{L}=\left.\hat{\mathcal{L}}\right|_{\mathcal{M}}+\delta \mathcal{L}
$$

Here, $\left.\hat{\mathcal{L}}\right|_{\mathcal{M}}$ is obtained by restricting $\hat{\mathcal{L}}$ to the subspace $\mathcal{M} \subset \hat{\mathcal{M}}$ (which corresponds to simply truncating out two vector multiplets), and $\delta \mathcal{L}$ contains the effective interactions generated by loops involving the two vector multiplets one integrates out.

Note that our problem is actually the reverse: we know $\mathcal{L}$, which is given by the polynomial $\mathcal{V}=S T U+\frac{1}{3} U^{3}$, and eventually want to reconstruct $\hat{\mathcal{L}}$. This is done by first determining $\left.\hat{\mathcal{L}}\right|_{\mathcal{M}}$ and then by fixing the undetermined parameters $\kappa$ and $\lambda$ in $\hat{\mathcal{L}}$ such that restricting $\hat{\mathcal{L}}$ to $\mathcal{M} \subset \hat{\mathcal{M}}$ gives $\left.\hat{\mathcal{L}}\right|_{\mathcal{M}}$. (At this stage it is not clear whether this second step is uniquely possible; it could be that the embedding of $\left.\hat{\mathcal{L}}\right|_{\mathcal{M}}$ into $\hat{\mathcal{L}}$ leaves room for an ambiguity in the parameters $\kappa$ and $\lambda$.)

To take the first step, we need to find $\delta \mathcal{L}$. This problem is simplified by the fact that the whole Lagrangian is determined by the coefficients $C_{I J K}$. The only effect of integrating out charged multiplets is a discrete change of these coefficients. In order to trace this, it is sufficient to look at the Chern-Simons term.

The precise form of the induced Chern-Simons term was computed in [7] for the case when a single charged hypermultiplet is integrated out. In [8] this was generalized to the case where both charged vector multiplets and charged hypermultiplets are integrated out, with the charged hypermultiplets in arbitrary representations of the gauge group. We will only need the case when vector multiplets and hypermultiplets are in the same representation (ultimately, the adjoint of $S U(2)$ ). Without loss of generality we can parameterize the gauge fields such that the charged states only couple to $A_{\mu}^{\star}$, where $\star$ is one particular value in the range of $I$.

The result is most conveniently expressed in terms of the corresponding prepotentials. If $\mathcal{V}=\left.\hat{\mathcal{V}}\right|_{\mathcal{M}}+\delta \mathcal{V}$, then, in our conventions,

$$
\delta \mathcal{V}=\frac{1}{2}\left(N_{H}-N_{V}\right)\left(h^{\star}\right)^{3},
$$

where $N_{H}$ and $N_{V}$ are the numbers of hypermultiplets and vector multiplets charged under $A_{\mu}^{\star}$, and $h^{\star}$ denotes the corresponding embedding coordinate. Compared to [7, 6, 5] the above and various of the following formulae differ by a factor $\frac{1}{6}$. This is due to our different normalization of the scalar fields. 


\subsection{The embedding}

We now apply this general result to our case, where $N_{H}=0, N_{V}=2$ and $h^{\star}=h^{1}=$ $6^{-1 / 3}(T-U)$ and obtain

$$
\left.\hat{\mathcal{V}}\right|_{\mathcal{M}}=S T U+\frac{1}{3} U^{3}+\frac{1}{6}(T-U)^{3}
$$

As indicated by our notation, this polynomial should now be the one that one obtains from (3.21) by a suitable restriction to the three variables $S, T, U$. More precisely, expressing $\hat{h}^{0}, \ldots, \hat{h}^{4}$ as appropriate linear combinations of $S, T, U$, the polynomial $\hat{\mathcal{V}}$ (eq. (3.21)) should reduce to (3.25). By "appropriate" linear combinations, we mean that $T=U$ should imply $S U(2)$ invariance, i.e., $\hat{h}^{1}=\hat{h}^{2}=\hat{h}^{3}=0$. This results in a system of ten coupled cubic equations for the coefficients appearing in these linear combinations (see Appendix A). Remarkably, a solution of this system exists if and only if the coefficient in front of $(T-U)^{3}$ in (3.25) is really $\frac{1}{6}$, i.e., exactly as the integrating out procedure suggests. For all other values of this coefficient, no solution exists. This reconfirms the importance of properly taking into account the one-loop threshold effects and is an important consistency check.

Taking now the right coefficient $\frac{1}{6}$, one further finds (see Appendix A) that $\hat{h}^{0}, \ldots, \hat{h}^{4}$ can be consistently expressed in terms of $S, T, U$ if and only if the coefficients $\kappa$ and $\lambda$ satisfy the constraints

$$
\lambda=\frac{1}{\sqrt{2}}, \quad \kappa>-\lambda, \quad \kappa \neq 2 \lambda .
$$

Note that the two excluded boundary values of $\kappa$, namely $\kappa=-\frac{1}{\sqrt{2}}$ and $\kappa=\sqrt{2}$ are precisely those values for which $\hat{\mathcal{M}}$ would have been a symmetric space.

It now seems that our embedding problem does have a consistent solution, but that this solution is not unique. In fact, there seems to be a one-parameter family of Lagrangians $\hat{\mathcal{L}}$ parameterized by the allowed values of $\kappa$ in the underlying cubic polynomial $\hat{\mathcal{V}}$. Surprisingly, this is not true. As is shown in Appendix B, every pair of admissible values $\kappa_{1}, \kappa_{2}$ can be transformed into each other by a linear redefinition of the $\hat{h}^{I}$. Therefore all these manifolds are physically equivalent, and the embedding is unique. The unambiguous construction of this supergravity theory from the underlying Calabi-Yau data is the main result of this paper.

For the sake of completeness, we note the explicit relations between $S, T, U$ and $\hat{h}^{I}$ :

$$
\begin{aligned}
\hat{h}^{0} & =\frac{1}{3 G^{2}}\left(\frac{2 U-T}{3}+S\right)+\frac{G}{3}(T+U) \\
\left(\hat{h}^{1}\right)^{2}+\left(\hat{h}^{2}\right)^{2}+\left(\hat{h}^{3}\right)^{2} & =\left(\frac{G^{2}}{6}-\frac{2}{9 G}\right)(T-U)^{2} \\
\hat{h}^{4} & =\frac{\sqrt{2}}{3}\left(\frac{2 U-T}{3 G^{2}}+\frac{S}{G^{2}}-\frac{G}{2}(T+U)\right),
\end{aligned}
$$

where

$$
G^{3}=-\frac{4}{3}\left(\frac{1+\sqrt{2} \kappa}{2-\sqrt{2} \kappa}\right) .
$$


Observe that $(T-U)^{2}$ is related to the gauge invariant combination $\left(\hat{h}^{1}\right)^{2}+\left(\hat{h}^{2}\right)^{2}+\left(\hat{h}^{3}\right)^{2}$. By a gauge transformation we can set $\hat{h}^{3} \propto T-U$, and then the truncation from $\hat{\mathcal{M}}$ to $\mathcal{M}$ is given by $\hat{h}^{1}=\hat{h}^{2}=0$.

\subsection{The Higgs effect}

The purpose of this subsection is to explicitly recover the expected Higgs effect in the $S U(2)$ gauged supergravity theory based on $\hat{\mathcal{L}}{ }^{2}$

Due to the absence of a scalar potential, any vev $\left\langle\phi^{x}\right\rangle$ of the scalar fields $\phi^{x}$ gives rise to a Minkowski ground state of the theory, which can be easily verified to preserve the full $\mathcal{N}=2$ supersymmetry [15] (provided that $\left\langle A_{\mu}^{I}\right\rangle=\left\langle\lambda^{i x}\right\rangle=\left\langle\psi_{\mu}^{i}\right\rangle=0$ ).

Any vev $\left\langle\phi^{x}\right\rangle$ of $\phi^{x}$ also determines a vev $\left\langle\hat{h}^{I}\right\rangle$ of the corresponding embedding coordinates. The vacua with $\left\langle\hat{h}^{A}\right\rangle=0, A=1,2,3$, leave the full $S U(2)$ gauge symmetry unbroken, whereas a non-vanishing value for at least one of the three $\left\langle\hat{h}^{A}\right\rangle$ spontaneously breaks $S U(2)$ to $U(1)$. In the latter case, the Calabi-Yau picture requires two vector multiplets to become massive BPS vector multiplets, in which the former scalar fields $\phi$ contribute the longitudinal modes of the massive 'W-bosons'.

If we choose $\left\langle\hat{h}^{3}\right\rangle$ to be the only non-vanishing vev of $\hat{h}^{A}$, the vector fields $A_{\mu}^{1,2}$ and the gaugini $\lambda^{i 1,2}$ should therefore acquire a mass proportional to $\left\langle\hat{h}^{3}\right\rangle$ (see eq. $(2.20)$ ).

To see how this happens, consider

$$
\begin{array}{ll}
A_{\mu}^{0}:=\left\langle\hat{h}_{I}\right\rangle A_{\mu}^{I}, & F_{\mu \nu}^{0}=\left\langle\hat{h}_{I}\right\rangle F_{\mu \nu}^{I} \\
A_{\mu}^{x}:=\left\langle\hat{h}_{I}^{x}\right\rangle A_{\mu}^{I}, & F_{\mu \nu}^{x}=\left\langle\hat{h}_{I}^{x}\right\rangle F_{\mu \nu}^{I},
\end{array}
$$

where $\hat{h}_{I}$ is as in eq. (2.16), and $\hat{h}_{I}^{x}$ is defined by 14

$$
\hat{h}_{I}^{x}:=\sqrt{\frac{3}{2}} g^{x y} \frac{\partial \hat{h}_{I}}{\partial \phi^{y}}
$$

In a given vacuum, the vector fields $A_{\mu}^{x}$ are the superpartners of $\lambda^{i x}$ and $\phi^{x}$, whereas $A_{\mu}^{0}$ is the graviphoton (i.e., $A_{\mu}^{x}$ and $A_{\mu}^{0}$ appear in the supersymmetry transformations of, respectively, the gaugini and the gravitini 150).

In order to see which of these vector fields acquires a mass, one uses the identity 14

$$
\stackrel{\circ}{a}_{I J}=\hat{h}_{I} \hat{h}_{J}+g_{x y} \hat{h}_{I}^{x} \hat{h}_{J}^{y}
$$

to rewrite the kinetic term of the vector fields in a given vacuum:

$$
e^{-1} \hat{\mathcal{L}}_{\mathrm{vec}}=-\frac{1}{4}\left\langle a_{I J}\right\rangle F_{\mu \nu}^{I} F^{\mu \nu J}=-\frac{1}{4} F_{\mu \nu}^{0} F^{\mu \nu 0}-\frac{1}{4}\left\langle g_{x y}\right\rangle F_{\mu \nu}^{x} F^{\mu \nu y} .
$$

Mass terms for these vector fields can only come from the kinetic term of the scalar fields,

$$
e^{-1} \hat{\mathcal{L}}_{\text {scalar }}=-\frac{1}{2} g_{x y} \mathcal{D}_{\mu} \phi^{x} \mathcal{D}^{\mu} \phi^{y}
$$

\footnotetext{
${ }^{2}$ In order to simplify the notation, we will not put hats on the fields appearing in $\hat{\mathcal{L}}$.
} 
where $\mathcal{D}_{\mu}$ denotes the $S U(2)$ covariant derivative of the scalar fields [15],

$$
\mathcal{D}_{\mu} \phi^{x}=\partial_{\mu} \phi^{x}+g A_{\mu}^{A} K_{A}^{x} .
$$

Using some identities of very special geometry [14, 15], one can show that the term quadratic in the vector fields in (3.36) can be brought to the form

$$
\frac{1}{2}\left(A_{\mu}^{x} A^{\mu y}\right)\left(g W_{x z}\right)\left(g W_{y}^{z}\right)
$$

where $W_{x y}:=h_{[x}^{A} K_{A y]}$.

Given the kinetic term for the gaugini (see [15])

$$
-\frac{1}{2}\left(\bar{\lambda}^{i x} \Gamma^{\mu} \nabla_{\mu} \lambda_{i}^{y}\right) g_{x y}
$$

and the Yukawa term (3.8) required by supersymmetry,

$$
e^{-1} \hat{\mathcal{L}}_{\text {Yuk }}=\frac{i}{2}\left(\bar{\lambda}^{i x} \lambda_{i}^{y}\right)\left(g W_{x y}\right)
$$

it is now easy to see that, in a given vacuum with a given vev $\left\langle W_{x y}\right\rangle, A_{\mu}^{x}$ and $\lambda^{i x}$ automatically have the same mass, and that the graviphoton $A_{\mu}^{0}$ remains massless, as it should. These observations are of course automatic consequences of the unbroken $\mathcal{N}=2$ supersymmetry of these vacua.

What remains to be verified is whether a non-vanishing vev $\left\langle\hat{h}^{3}\right\rangle$ really introduces a mass proportional to $\left\langle\hat{h}^{3}\right\rangle$ for $A_{\mu}^{1,2}$ and $\lambda^{i 1,2}$, and whether all other fields remain massless in such a vacuum. According to what we have said above, it is sufficient to look at the masses of the fermions $\lambda^{i x}$.

To this end, we choose the 'special coordinates'

$$
\phi^{x}=\frac{\hat{h}^{x}}{\hat{h}^{0}}, \quad x=1, \ldots, 4
$$

to parameterize the scalar manifold $\hat{\mathcal{M}}$. This choice allows one to explicitly solve $\hat{h}^{I}$ as functions of the $\phi^{x}$ using the constraint $\hat{\mathcal{V}}=1$.

For the Yukawa term, one then obtains

$$
e^{-1} \hat{\mathcal{L}}_{\mathrm{Yuk}}=\frac{i}{2} \bar{\lambda}^{i A} \lambda_{i}^{B}\left[\left(\frac{3}{2}\right)^{3 / 2} g\left(1+\kappa \phi^{4}\right)\left(\hat{h}^{0}\right)^{3}\right] \epsilon_{A B C} \hat{h}^{C} .
$$

We will now allow $\left\langle\hat{h}^{3}\right\rangle$ and $\left\langle\hat{h}^{4}\right\rangle$ to be non-zero, whereas $\left\langle\hat{h}^{1}\right\rangle$ and $\left\langle\hat{h}^{2}\right\rangle$ are assumed to vanish.

The vev of the mass matrix $W_{x y}$ is then

$$
\left\langle W_{x y}\right\rangle=\left(\begin{array}{cccc}
0 & a & 0 & 0 \\
-a & 0 & 0 & 0 \\
0 & 0 & 0 & 0 \\
0 & 0 & 0 & 0
\end{array}\right),
$$


where $a=\left(\frac{3}{2}\right)^{\frac{3}{2}}\left(1+\kappa\left\langle\phi^{4}\right\rangle\right)\left\langle\hat{h}^{0}\right\rangle^{3}\left\langle\hat{h}^{3}\right\rangle$.

In order to really extract the physical masses from this matrix, one has to make sure that one works in a field basis with canonically normalized kinetic terms.

In our class of vacua, where $\left\langle\hat{h}^{1}\right\rangle=\left\langle\hat{h}^{2}\right\rangle=0$, the vev of the metric $g_{x y}$ turns out to be of the form

$$
\left\langle g_{x y}\right\rangle=\left(\begin{array}{cccc}
b & 0 & 0 & 0 \\
0 & b & 0 & 0 \\
0 & 0 & \left\langle g_{33}\right\rangle & \left\langle g_{34}\right\rangle \\
0 & 0 & \left\langle g_{43}\right\rangle & \left\langle g_{44}\right\rangle
\end{array}\right)
$$

with $b:=\frac{3}{2}\left(1+\kappa\left\langle\phi^{4}\right\rangle\right)\left\langle\hat{h}^{0}\right\rangle^{3}$

In order to obtain canonically normalized $\lambda^{i x}$, one has to redefine the $\lambda^{i x} \rightarrow \lambda^{\prime i x}$ such that $\bar{\lambda}^{i x} \lambda_{i}^{y}\left\langle g_{x y}\right\rangle=\bar{\lambda}^{i x} \lambda_{i}^{\prime y} \delta_{x y}$. Due to the peculiar form of $\left\langle g_{x y}\right\rangle, \lambda^{\prime i 3}$ and $\lambda^{\prime i 4}$ will be some linear combinations of $\lambda^{i 3}$ and $\lambda^{i 4}$, whereas $\lambda^{\prime i 1}$ and $\lambda^{i 2}$ are obtained by a mere rescaling,

$$
\lambda^{\prime i 1,2}=b^{\frac{1}{2}} \lambda^{i 1,2}
$$

In terms of these new, canonically normalized gaugini (whose primes we will omit from now on), the Yukawa term becomes

$$
\hat{\mathcal{L}}_{\text {Yuk }}^{\text {can }}=\frac{i}{2}\left(\bar{\lambda}^{i 1} \lambda_{i}^{2}-\bar{\lambda}^{i 2} \lambda_{i}^{1}\right) Q\left\langle\hat{h}^{3}\right\rangle
$$

with $Q=\sqrt{\frac{3}{2}} g$. This shows that only the gaugini $\lambda^{i 1,2}$ (and with them the vector fields $A_{\mu}^{1,2}$ ) acquire a mass, and that this mass is indeed proportional to $\left\langle\hat{h}^{3}\right\rangle$,

$$
m_{\lambda^{1,2}}=Q\left\langle\hat{h}^{3}\right\rangle
$$

Since the charge of a BPS M2-brane is proportional to its tension, the $S U(2)$ gauge coupling $g$ must be equal to $T_{(2)}$, up to a constant which we do not attempt to compute here.

\section{Elementary transformations, reflected cones and the Weyl twist}

In the extended scalar manifold $\hat{\mathcal{M}}$ there is no obstruction in going to negative values of $\hat{h}^{3} \propto T-U$, whereas in the original model $T=U$ is the boundary of the Kähler cone, and a continuation to negative $T-U$ seems to be impossible because the Calabi-Yau space becomes singular at $T=U$. How can these two facts be reconciled?

As in many similar cases, it makes sense to continue the theory beyond the boundary of the Kähler cone. In general such continuations involve a change of topology. The basic mechanism is that the singular points of the singular manifold $\hat{X}$ can be resolved in two different ways, thus giving rise to two different families $X, \tilde{X}$ of smooth Calabi-Yau spaces. 
As will become more and more clear during this section, the singular space $\hat{X}$ has a close relation to the gauged Lagrangian $\hat{\mathcal{L}}$. This motivates our notation.

The Kähler cones of the two families $X, \tilde{X}$ can be glued together along the face corresponding to the singular manifold $\hat{X}$. One example, where $X$ and $\tilde{X}$ have different topology (triple intersection numbers) but are still birationally equivalent are flop transitions [7]. The case we are considering, on the other hand, corresponds to an elementary transformation. In this case the families $X, \tilde{X}$ are biholomorphically equivalent. It will turn out that the symmetry relating $X$ to $\tilde{X}$ is nothing but an $S U(2)$ gauge transformation.

In Section 4.1 we will review some general facts about topological phase transitions in Calabi-Yau threefolds, then, in Section 4.2 we consider the elementary transformation occuring in our models explicitly. In a little digression we discuss the relevance of elementary transformations and reflected cones for space-time geometries where $S U(2)$ gauge symmetry enhancement occurs at special points in space-time. Finally, we show in Section 4.3 how the elementary transformation is realized in the $S U(2)$-gauged effective supergravity that includes the additional light modes.

\subsection{General discussion of elementary transformations}

This subsection is a short review of material from [7, 5].

Let $h^{I}>0$ parameterize the Kähler cone of a Calabi-Yau threefold $X$ and let $h^{\star}=0$ be the boundary where the two-cycle $C^{\star}$ collapses. ( $\star$ is just one of the possible values of $I$.) Thus at the boundary $h^{\star}=0$ the volume of $C^{\star}$,

$$
\operatorname{vol}\left(C^{\star}\right)=\int_{C^{\star}} J=h^{\star}
$$

vanishes and it becomes negative when one continues naively to negative $h^{\star}$. Correspondingly, an M2-brane wrapped on $C^{\star}$ seems to get a negative mass

$$
M=T_{(2)} h^{\star}
$$

for negative $h^{\star}$.

But, as already explained above, the range of negative $h^{\star}$ actually corresponds to a different Calabi-Yau space $\tilde{X}$, which is obtained by resolving the singularities of $\hat{X}$ in a different way, and which in general has a topology different from the one of $X$.

In the most general topological transitions the Hodge numbers, and with them the numbers of neutral vector and hypermultiplets [2, 4, 5], change. This requires the presence of at least two adjoint hypermultiplets (or of hypermultiplets in other representations of the gauge group) at the singularity, so that the scalar potential has both a Coulomb and a Higgs

branch. In our case these necessary conditions are not satisfied, and the Hodge numbers of $X$ and $\tilde{X}$ are the same. Even if the Hodge numbers do not change, the manifolds $X$ and $\tilde{X}$ might still have different topology. If the triple intersection numbers are different and are not related by a basis transformation of the Kähler cone, then $X$ and $\tilde{X}$ have different topology. But if the intersection numbers are equivalent, then $X$ and $\tilde{X}$ have the same topology, and 
the transformation relating them is an isomorphism (in fact a gauge transformation as we will see below). In both cases one can glue together the Kähler cones of $X$ and $\tilde{X}$ along the face $h^{\star}=0$. Within the Kähler cone of $\tilde{X}$ the cycle $C^{\star}$ is replaced by $\tilde{C}^{\star}=-C^{\star}$, which has positive volume $\tilde{h}^{\star}=-h^{\star}$ when measured with the Kähler form $\tilde{J}$ of $\tilde{X}$. When one goes from positive to negative $h^{\star}$, states obtained from wrapping an M2-brane on $C^{\star}$ become massless and then get a positive mass again.

The explicit transformation relating $X$ to $\tilde{X}$ depends on the details of the degeneracy at $h^{\star}=0$. For proper transformations (flops) and elementary transformations it can be computed using methods from algebraic geometry. Alternatively, one can analyze the additional massless modes present at $h^{\star}=0$ and compute the change induced in the prepotential by integrating them out [7, 6]. The result of this depends on whether $h^{\star}>0$ or $h^{\star}<0$. These two cases differ in the sign of the mass of the charged particles running in the loops (where mass is defined from the point of view of manifold $X$, i.e., it becomes negative for $h^{\star}<0$ ). Using the notation of Section 3, integrating out charged states at $h^{\star}>0$ gives

$$
\mathcal{V}=\left.\hat{\mathcal{V}}\right|_{\mathcal{M}}+\delta \mathcal{V}
$$

whereas the result for $h^{\star}<0$ is

$$
\tilde{\mathcal{V}}=\left.\hat{\mathcal{V}}\right|_{\mathcal{M}}-\delta \mathcal{V}
$$

Using (3.24) one finds the following discontinuity at $h^{\star}=0$ :

$$
\tilde{\mathcal{V}}-\mathcal{V}=-2 \delta \mathcal{V}=\left(N_{V}-N_{H}\right)\left(h^{\star}\right)^{3}
$$

This discontinuity is a five-dimensional one-loop threshold effect [19, 7, 6], analogous to the well-known logarithmic singularity of the prepotential in four dimensions [32, 2]. Since in five dimensions the whole vector multiplet sector of the effective theory of uncharged massless modes is determined by the cubic prepotential, a discrete jump of its coefficients (the triple intersection numbers) is the only possible threshold effect.

This field theory calculation can be compared to the formulae for proper and elementary transformations obtained in algebraic geometry:

(i) Take the case $N_{H}=1, N_{V}=0$ of a single massless hypermultiplet. In this case one obtains

$$
\tilde{\mathcal{V}}-\mathcal{V}=-\left(h^{\star}\right)^{3}
$$

which is the correct formula for a flop transition, where $X$ and $\tilde{X}$ are related by a proper transformation [7]. More generally, there are transitions where $N_{H}$ curves are flopped, and then $\tilde{\mathcal{V}}-\mathcal{V}=-N_{H}\left(h^{\star}\right)^{3}$.

(ii) The case $N_{H}=2 g, N_{V}=2$ of $S U(2)$ gauge symmetry enhancement with $g$ adjoint hypermultiplets. Geometrically this is realized by the collapse of a divisor $E$ into a genus $g$ curve of $A_{1}$ singularities. The supergravity formula gives

$$
\tilde{\mathcal{V}}-\mathcal{V}=(2-2 g)\left(h^{\star}\right)^{3}
$$


which coincides with the geometrical formula for an elementary transformation relating $X$ and $\tilde{X}$ [5].

Whereas proper transformations relate manifolds with different topology, $X$ and $\tilde{X}$ are isomorphic (biholomorphically equivalent) for elementary transformations. This is obvious since the elementary transformation acts on the Kähler cone by a reflection, which could be reinterpreted as a change of basis in $H_{2}(X, \mathbb{Z})$. We will see this explicitly in Section 4.2, and in Section 4.3 we will show that the basis transformation is an $S U(2)$ gauge transformation. In this case the Kähler cone of $\tilde{X}$ is called the reflected cone. One might wonder whether gluing in the reflected Kähler cone has any use, because the original Kähler cone already covers all inequivalent manifolds once. It turns out that working with a doubled range of variables is nevertheless useful for several reasons:

(i) Working with the reflected cone is useful for studying solutions which dynamically run into $S U(2)$ gauge symmetry enhancement. This will be briefly explained in the next subsection and covered in detail in [33.

(ii) It is instructive to see what the elementary transformation is in terms of gauged supergravity. As we will see in Section 4.3, this makes use of the reflected Kähler cone.

(iii) If the genus of the curve of $A_{1}$ singularities is $g>0$, then not only charged vector multiplets but also charged hypermultiplets become massless. When taking into account deformations of the complex structure, which are hypermultiplet moduli, one finds that the elementary transformation acts on both kinds of multiplets in such a way that the factorization of the moduli space into vector multiplet moduli and hypermultiplet moduli breaks down[5]. The point of enhanced symmetry is an orbifold point of the combined moduli space and one needs to introduce the reflected cone to describe the moduli space properly. If at least two adjoint hypermultiplets are present, then there exist extremal transitions which change the Hodge numbers of $X$, 击, 5, 6]. This corresponds to a scalar potential which possesses both a Coulomb and a Higgs branch.

\subsection{Application to our models and consequences for space-time geometries}

The case we have been studying in this paper is $N_{H}=2 g=0, N_{V}=2$. Switching from the variables $h^{I}$, which are adapted to the Kähler cone, to the variables $S, T, U$ and using the general formulae (4.3) and (4.4), we find the following prepotentials associated to $X$ and $\tilde{X}$ :

$$
\begin{aligned}
\mathcal{V}=S T U+\frac{1}{3} U^{3} & \text { for } T>U \\
\tilde{\mathcal{V}}=S T U+\frac{1}{3} U^{3}+\frac{1}{3}(T-U)^{3} & \text { for } T<U
\end{aligned}
$$

Note that one could make a basis transformation and bring the prepotential for $T<U$ to the

form $\tilde{\mathcal{V}}=S T U+\frac{1}{3} T^{3}$ 19. But when working with the combined cones of $X$ and $\tilde{X}$ one has to apply the same change of basis to $\mathcal{V}$, which then no longer is of the form $S T U+\frac{1}{3} U^{3}$. This has important consequences for space-time geometries with rolling moduli. When considering 
backgrounds where $h^{\star}$ starts at a positive value and dynamically evolves to zero, one has to study whether and how such solutions can be continued to $h^{\star}<0$. This problem was analyzed for domain walls in the model with $X=X_{1}$ in [34]. Since both the metric $g_{x y}$ on the moduli space and the space-time metric describing the domain wall were found to be smooth, it was expected that one could continue the solution using the method introduced in [35]. But in [34] the prepotential used to define the continuation was $\mathcal{V}=S T U+\frac{1}{3} U^{3}$ for $T>U$ and $\tilde{\mathcal{V}}=S T U+\frac{1}{3} T^{3}$ for $T<U$. Using this rule for the continuation, it was found that the space-time Riemann tensor of the domain wall solution has $\delta$-function singularities at the space-time points at which $T=U$. In other words, one would have to introduce a localized source of stress-energy at the points in space where $T=U$.

This is different when one uses the correct continuation of the prepotential which we obtained above by properly applying an elementary transformation. It can be shown that there is no such $\delta$-function singularity, but instead one finds that the space-time metric is $C^{2}$, precisely as in flop transitions occuring dynamically in black hole geometries [30, 35] and domain walls [36]. In fact one can show that continuing black hole, black string or domain wall solutions through an $S U(2)$ boundary is equivalent to reflecting it at the boundary by a gauge transformation. Using this one can prove that for the models based on the CalabiYau threefolds $X_{0}, X_{1}, X_{2}$ no space-time singularities can occur as long as the moduli roll within the extended Kähler cone. In particular, all apparent space-time singularities found in supergravity are not really present in M-theory, because the $S U(2)$ enhancement modifies the evolution of the solution. This explains how the new mechanism for excising space-time singularities proposed in [34] works. We refer to a future publication for the details [33].

We now turn to an explicit description of how $\tilde{X}$ is obtained from $X$. In the next section we will show that this transformation is an $S U(2)$ gauge transformation.

To construct $\tilde{X}$ from $X$, we need to specify how the prepotential (triple-intersection numbers) and the boundaries of the Kähler cones are related. Let $C^{I}$ and $D_{I}$ again be the standard generators of $H_{2}(X, \mathbb{Z})$ and $H_{4}(X, \mathbb{Z})$.

There is a family of holomorphic curves $\Gamma$ which sweeps out a divisor $E$. The elementary transformation acts by [5]

$$
D_{I} \longrightarrow \tilde{D}_{I}=D_{I}+\left(D_{I} \cdot \Gamma\right) E
$$

Since $\Gamma \cdot E=-2$ [5] one has $E \rightarrow-E$, showing that the elementary transformation acts as a reflection. Therefore the Kähler cone of $\tilde{X}$ is called the reflected cone. The collection of all cones obtained by reflections is called the reflected movable cone [5].

One can now compute $\tilde{C}_{I J K}$, and the new dual two-cycles $\tilde{C}^{I}$ are determined from $\tilde{C}^{I}$. $\tilde{D}_{J}=\delta_{J}^{I}$.

In our models the $S U(2)$ enhancement occurs for $h^{1}=0$. This means that the curves $\Gamma$ are in the homology class $C^{1}$. The dual four-cycles $D_{I}$ are determined by the dual embedding coordinates $h_{I}=C_{I J K} h^{J} h^{K}$. We have to identify a four-cycle which collapses to a two-cycle for $h^{1}=0$. This identifies the homology class of the divisor $E$. Since the intersection numbers enter, this cycle is different for the three models $X_{0}, X_{1}, X_{2}$. To be a bit more explicit, we 
consider the model $X_{1}$. There we have

$$
\begin{aligned}
& h_{0}=8\left(h^{0}\right)^{2}+6 h^{0} h^{1}+\left(h^{1}\right)^{2}+4 h^{0} h^{2}+2 h^{1} h^{2} \\
& h_{1}=3\left(h^{0}\right)^{2}+2 h^{0} h^{1}+2 h^{0} h^{2} \\
& h_{2}=2\left(h^{0}\right)^{2}+2 h^{0} h^{1} .
\end{aligned}
$$

There is a unique linear combination (up to an overall constant) which vanishes for $h^{1}=0$ :

$$
h=h_{0}-2 h_{1}-h_{2}=h^{1}\left(h^{1}+h^{2}\right) .
$$

Note that $h$ goes to zero $\sim h^{1}$, indicating that the four-cycle collapses to a two-cycle rather than to a zero-cycle. Thus we have in homology

$$
E=D_{0}-2 D_{1}-D_{2}
$$

Observe that $C^{1} \cdot E=-2$ as it must. Now applying formula (4.10) for an elementary transformation, one obtains the new four-cycles

$$
\tilde{D}_{0}=D_{0}, \quad \tilde{D}_{1}=D_{0}-D_{1}-D_{2}, \quad \tilde{D}_{2}=D_{2}
$$

and the new dual two-cycles are

$$
\tilde{C}^{0}=C^{0}+C^{1}, \quad \tilde{C}^{1}=-C^{1}, \quad \tilde{C}^{2}=-C^{1}+C^{2} .
$$

Now we can read off the transformation of the $h^{I}$ :

$$
h^{0} \rightarrow h^{0}+h^{1}, \quad h^{1} \rightarrow-h^{1}, \quad h^{2} \rightarrow-h^{1}+h^{2} .
$$

Converting this to $S, T, U$ we find

$$
T \leftrightarrow U \quad S \rightarrow S+U-T
$$

\subsection{The Weyl twist}

Given that the elementary transformation acts as a $\mathbb{Z}_{2}$ reflection, the natural explanation in terms of the gauge theory is to identify it with the Weyl twist of $S U(2)$ [5, 6].

Recall that the Weyl group of a simple Lie group $G$ consists of all inner automorphisms that leave the maximal torus invariant modulo those which do so pointwise:

$$
W(G)=N(T) / T
$$

where $W(G)$ is the Weyl group of $G$, and $N(T)$ is the normalizer of the maximal torus $T$. The Weyl group of $S U(2)$ is isomorphic to $\mathbb{Z}_{2}$, its generator is called the Weyl twist.

In our $S U(2)$ gauged supergravity theory we have fields $\hat{h}^{A}$ in the adjoint and singlets $\hat{h}^{0}, \hat{h}^{4}$. The singlets are of course invariant. Taking the maximal torus to be generated by $\hat{h}^{3}$, the Weyl twist acts by $\hat{h}^{3} \rightarrow-\hat{h}^{3}$, while all other fields are invariant. Now we make use 
of the unique solution of the embedding problem we found in Section 3. Using the explicit relations (3.27) - (3.29) between the variables $\hat{h}^{I}$ and the variables $S, T, U$ we immediately find that $S, T, U$ precisely transform as in (4.19). This shows that the Weyl twist operates as an elementary transformation on the Calabi-Yau spaces.

It is also instructive to consider the behaviour of the prepotentials $\mathcal{V}, \hat{\mathcal{V}}, \tilde{\mathcal{V}}$ under the Weyl twist. By construction, the prepotential $\hat{\mathcal{V}}$ is invariant. This is still true after projecting to $\mathcal{M}$, i.e.,

$$
\left.\hat{\mathcal{V}}\right|_{\mathcal{M}}=S T U+\frac{1}{3} U^{3}+\frac{1}{6}(T-U)^{3}
$$

is invariant under (4.19). The prepotentials $\mathcal{V}$ and $\tilde{\mathcal{V}}$ that we obtained by integrating out the charged vector multiplets for $T-U>0$ and $T-U<0$ are not invariant but are precisely mapped to one another under (4.19). This shows explicitly that the models defined by compactification on $X$ and $\tilde{X}$ are related by an $S U(2)$ gauge transformation.

It is interesting to ask what interpretation the full prepotential $\hat{\mathcal{V}}$ might have in terms of Calabi-Yau geometry. Its coefficients are not intersection numbers of either $X$ or $\tilde{X}$, but $\left.\hat{\mathcal{V}}\right|_{\mathcal{M}}$ is obtained as a 'superposition' or 'orbit sum':

$$
\left.\hat{\mathcal{V}}\right|_{\mathcal{M}}=\frac{1}{2}(\mathcal{V}+\tilde{\mathcal{V}})
$$

while the extension to the full $\hat{\mathcal{V}}$ seems to be dictated by gauge symmetry (this is at least what we realized a posteriori above).

The above relation is true more generally for integrating out $N_{H}$ hypermultiplets and $N_{V}$ vector multiplets, even though in those cases the $\mathbb{Z}_{2}$ need not be a symmetry transformation. In particular, it is not a symmetry transformtion for flops. The natural conjecture is that $\hat{\mathcal{V}}$ plays a rôle in the intersection theory of singular Calabi-Yau spaces. For example the coefficients $\hat{C}_{I J K} I, J, K=0, \ldots, 4$ could be used to construct a generalization of the (co)homology ring defined by $C_{I J K}, I, J, K=0,1,2$. Gauge theory and gauged supergravity might provide the tool to describe singular Calabi-Yau spaces as regular objects, presumably by adding extra data (corresponding to wrapped branes) located at the vanishing cycles.

The transformation (4.19) is natural from the point of view of the dual heterotic string. There, the transformation $T \leftrightarrow U$ corresponds to inverting the radius $R \rightarrow \frac{\alpha^{\prime}}{R}$ [19. At the fixed point $T=U \Leftrightarrow R=\sqrt{\alpha^{\prime}}$ of this transformation the Abelian Kaluza Klein gauge symmetry $U(1)$ is enhanced to $S U(2)$. This is one example of the interpretation of the Tduality group as a discrete gauge symmetry. For toroidal compactifications it has been shown [20] that the full T-duality is generated by Weyl twists of $S U(2)$ gauge groups that are unHiggsed at special loci of the moduli space. The fact that $S$, the heterotic dilaton, is not invariant under this transformation (see eq. (4.19)), as naively expected, is a typical one-loop effect in heterotic perturbation theory [32].

\section{Conclusions and outlook}

In this paper we have constructed the first explicit example of an effective gauged supergravity Lagrangian that incorporates extra light modes descending from branes wrapped on a 
vanishing cycle. We did this for a particular situation, $S U(2)$ gauge symmetry enhancement without charged matter in the compactification of M-theory on a Calabi-Yau threefold. From the duality to heterotic string theory on $K 3 \times S^{1}$, it follows that our results also apply to stringy perturbative mechanisms of gauge symmetry enhancement.

After solving a complicated algebraic embedding problem, we encountered a structure that is very rigid and seems to be completely determined by gauge symmetry. The first step of reversing the effects of integrating out charged multiplets results in a symmetrization of the prepotential $\mathcal{V}$ with respect to the transformation naturally associated with the singularity. In our examples these were either elementary transformations or proper transformations in the case of the flop. In both cases one gets the sum over orbits of the transformation,

$$
\left.\hat{\mathcal{V}}\right|_{\mathcal{M}}=\frac{1}{2}(\mathcal{V}+\tilde{\mathcal{V}})
$$

In the second step, which we only carried out for the specific case of $S U(2)$ gauge symmetry enhancement without additional matter, the re-installation of the extra mutiplets is achieved by replacing one variable, $\hat{h}^{3} \propto h^{1} \propto(T-U)$, by the appropriate invariant: $\left(\hat{h}^{3}\right)^{2} \rightarrow\left(\hat{h}^{1}\right)^{2}+$ $\left(\hat{h}^{2}\right)^{2}+\left(\hat{h}^{3}\right)^{2}$. We expect that a similar systematics is also present in more general cases.

The work presented here can be extended in various directions. One main direction is the systematic construction of supergravity Lagrangians from compactifications of M-theory on singular manifolds. Here M-theory is understood to include perturbative string compactifications, generalized string compactifications with fluxes and branes, as well as F-theory. We expect that the known relations between ungauged supergravity and smooth manifolds extend in a systematic way to gauged supergravity and singular manifolds. As we already mentioned, this might also be interesting from the mathematical point of view.

Some physical applications of such constructions were already mentioned in the introduction, but there are various other interesting aspects that might be worth studying. Compactifications on singular manifolds might for example provide an alternative tool for embedding certain supersymmetric brane world scenarios (such as [37, 38]) into string or M-theory. The bulk theories of many of these models typically involve certain types of $5 \mathrm{D}, \mathcal{N}=2$ gauged supergravity theories, and it is not always known how these can be embedded into M-theory. One well-understood mechanism for generating gauged supergravity theories from compactifications is to turn on background fluxes (see e.g. [39, 40] for the particular case of M-theory compactifications on Calabi-Yau threefolds. For an exhaustive list of references on more general Calabi-Yau compactifications with background fluxes see, e.g., [41]). While such background fluxes lead to rather generic gaugings of the R-symmetry group, the gaugings of scalar manifold isometries one obtains from this mechanism are rather non-generic. In this sense, compactifications on singular manifolds might be viewed as a complementary tool for generating gauged supergravities from compactifications. Natural further steps include the addition of hypermultiplets or compactifications on singular background manifolds in combination with fluxes.

It will also be very interesting to investigate four-dimensional $\mathcal{N}=2$ supergravity along the same lines. The simplest case to discuss is perturbative gauge symmetry enhancement in 
the heterotic string on $K 3 \times T^{2}$. A closely related and even more interesting case is its nonperturbative form, described by the conifold singularity in the dual type II compactification. Another possible extension is to consider higher rank non-Abelian gauge groups, which are unbroken on loci of higher codimension in the moduli space. A further, but more difficult step would of course be the extension to four-dimensional $\mathcal{N}=1$ compactifications.

In Section 4.2 we mentioned an example of an apparent space-time singularity in a solution of five-dimensional supergravity. This illustrates the second main direction of application and extension of the results of this paper: the study of space-time backgrounds where topological phase transistions or gauge symmetry enhancement are realized dynamically. By this we mean that in a background with space-time dependent moduli the moduli can evolve in such a way that they reach the special locus corresponding to gauge symmetry enhancement or topological transitions. This is closely related to the absence of space-time singularities in stringy backgrounds, because the new dynamics occuring at special points in moduli space are among the mechanisms which resolve or excise space-time singularities that are apparently present in a naive analysis based on (super)-gravity [42, 34].

Presently under investigation are BPS solutions of five-dimensional supergravity coupled to vector multiplets, where the moduli vary through the Kähler cone. This was already discussed in subsection 4.2, and the results will be presented in a forthcoming paper [33]. As an extension of this one would like to investigate the same solutions in the context of the full $S U(2)$ gauged supergravity theory constructed in this paper. While solutions in the ungauged effective theory are not smooth, but only $C^{2}$ at the points where the $S U(2)$ enhancement happens, one might expect that this is an artifact of integrating out massless supermultiplets and that the discontinuities are smoothed out when lifting the solutions to the full gauged supergravity. Whether this really happens is not clear to us at this point, but we expect that the comparison of the same backgound in terms of two different effective Lagrangians will be very instructive.

In [36], the effects of certain topological transitions within the setup of heterotic M-theory [43] were investigated, and it would be interesting to extend this analysis to more general types of transitions. A further extension along these lines could be a systematic study of time-dependent solutions, in particular those relevant for cosmology and the analysis of big bang and big crunch singularities (for a recent discussion see e.g. [44]).

\section{Acknowledgments}

We would like to thank J. Louis for many useful discussions. The interest of one of the authors [T.M.] in the work presented here resulted from inspiring discussions with S. Kachru, R. Kallosh, M. Shmakova and E. Silverstein on the results of [34]. M.Z. thanks J. Ellis and 
M. Günaydin for discussions on related material from [26]. We would further like to thank B. Szendrői for bringing ref. 45] to our attention, which discusses elementary transformations from the mathematical point of view. We also thank him for pointing out a terminological error in the first version of the present paper. Similarly, we thank W. Mück for contributing an elegant shortcut that considerably simplifies the first part of the calculation given in Appendix A.

\section{Appendix}

\section{A. The embedding}

This appendix contains some details on the reconstruction of the polynomial $\hat{\mathcal{V}}$.

Consider the polynomial

$$
\mathcal{V}(S, T, U)=S T U+\frac{1}{3} U^{3}+a(T-U)^{3}
$$

where $a$ is an as yet arbitrary constant (The original polynomials considered in Section 2.3 obviously corespond to $a=0$, whereas the version that takes into account the one-loop threshold effects (Section 3.3) would correspond to $a=1 / 6$. We leave this constant $a$ open at this point in order to see whether there are preferred values from the embedding point of view.)

In the following, we will use slightly different coordinates

$$
\begin{aligned}
& X=U \\
& Y=(T-U) \\
& Z=S
\end{aligned}
$$

in terms of which the polynomial (A.1) reads

$$
\mathcal{V}(X, Y, Z)=Z X^{2}+X Y Z+\frac{1}{3} X^{3}+a Y^{3}
$$

This polynomial describes a two-dimensional scalar manifold $\mathcal{M}$. The set of points given by $Y=0$ forms a codimension one hypersurface, i.e., a line, in $\mathcal{M}$.

Our goal now is to embed the two-dimensional scalar manifold $\mathcal{M}$ based on (A.5) into the four-dimensional scalar manifold $\hat{\mathcal{M}}$ based on the polynomial $\hat{\mathcal{V}}$ (see eq. (3.21)) such that the line $Y=0$ in $\mathcal{M}$ coincides with (at least part of) the line of $S U(2)$ invariant points in $\hat{\mathcal{M}}$.

We assume that this embedding can be achieved by simply embedding the corresponding polynomials into each other. 
Requiring that $Y=0$ implies $\hat{h}^{A}=0$ then leads to the most general ansatz (which has to be linear to keep the polynomial cubic)

$$
\begin{aligned}
& \hat{h}^{0}=A X+B Y+C Z \\
& \hat{h}^{1}=D Y \\
& \hat{h}^{2}=E Y \\
& \hat{h}^{3}=F Y \\
& \hat{h}^{4}=G X+H Y+I Z
\end{aligned}
$$

with $R^{2}:=D^{2}+E^{2}+F^{2} \neq 0$.

Inserting this into (3.21) and comparing with the coefficients of (A.5) leads to a coupled system of ten cubic equations for the coefficients $A, \ldots, I$ :

$$
\begin{aligned}
A^{3}-\frac{3}{2} A G^{2}+\lambda G^{3} & =\frac{1}{3} \\
B^{3}-\frac{3}{2} B H^{2}+\lambda H^{3}-\frac{3}{2} R^{2}[B+\kappa H] & =a \\
C^{3}-\frac{3}{2} C I^{2}+\lambda I^{3} & =0 \\
B\left[3 A^{2}-\frac{3}{2} G^{2}\right]+H\left[-3 A G+3 \lambda G^{2}\right] & =0 \\
C\left[3 A^{2}-\frac{3}{2} G^{2}\right]+I\left[-3 A G+3 \lambda G^{2}\right] & =1 \\
A\left[3 B^{2}-\frac{3}{2} R^{2}-\frac{3}{2} H^{2}\right]+G\left[-3 B H+3 \lambda H^{2}-\frac{3}{2} \kappa R^{2}\right] & =0 \\
C\left[3 B^{2}-\frac{3}{2} R^{2}-\frac{3}{2} H^{2}\right]+I\left[-3 B H+3 \lambda H^{2}-\frac{3}{2} \kappa R^{2}\right] & =0 \\
A\left[3 C^{2}-\frac{3}{2} I^{2}\right]+G\left[-3 C I+3 \lambda I^{2}\right] & =0 \\
B\left[3 C^{2}-\frac{3}{2} I^{2}\right]+H\left[-3 C I+3 \lambda I^{2}\right] & =0 \\
6 A B C-3[A H I+G I B+C G H]+6 \lambda G H I & =1
\end{aligned}
$$

These equations also involve the unknown coefficients $(\lambda, \kappa)$ as well as $a$. Being optimistic, one might therefore hope that a consistent solution to the above equations only exists for a small number of values for $(\lambda, \kappa)$ and $a$. We will now see to what extent this is the case. Our strategy is to first show that a consistent solution of (A.11)-A.20) can only exist if $\lambda^{2}=\frac{1}{2}$. As mentioned in Section 3.2, a redefinition $\hat{h}^{4} \rightarrow-\hat{h}^{4}$ can always be used to render $\lambda$ positive, i.e., we can always choose the positive root $\lambda=+\frac{1}{\sqrt{2}}$. Let us sketch how this result is obtained. ${ }^{3}$

\footnotetext{
${ }^{3}$ This more elegant derivation of $\lambda^{2}=\frac{1}{2}$ replaces a lengthier argument given in the first version of this paper and was contributed by W. Mück.
} 
First, rewrite eqs. (A.13) and (A.18) as

$$
\begin{aligned}
& C\left[C^{2}-\frac{1}{2} I^{2}\right]+I\left[-C I+\lambda I^{2}\right]=0 \\
& A\left[C^{2}-\frac{1}{2} I^{2}\right]+G\left[-C I+\lambda I^{2}\right]=0 .
\end{aligned}
$$

Viewing this as a linear system for $\left[C^{2}-\frac{1}{2} I^{2}\right]$ and $\left[-C I+\lambda I^{2}\right]$, one sees that either $\left[C^{2}-\frac{1}{2} I^{2}\right]=$ $\left[-C I+\lambda I^{2}\right]=0$, or $C G-A I=0$.

Let us first assume $C G-A I=0$. Multiplying (A.11) by $I^{3}$, one then derives $I=0$ by taking into account eq. (A.13). In a similar way, one derives $C=0$ by multiplying (A.11) by $C^{3}$. However, $C=I=0$ is a contradiction to (A.15). Hence, we have to assume the second possibility $\left[C^{2}-\frac{1}{2} I^{2}\right]=\left[-C I+\lambda I^{2}\right]=0$. But then, because $C=I=0$ is ruled out by (A.15), we immediately find the desired result $\lambda^{2}=\frac{1}{2}$.

To sum up, we have shown that if a consistent embedding exists, it can at most work with

$$
\lambda^{2}=\frac{1}{2}
$$

As mentioned above, there is no loss of generality in choosing the positive root

$$
\lambda=+\frac{1}{\sqrt{2}}
$$

as we will do from now on.

Note that we have never used (A.12) to arrive at this result. (A.12) is the only equation that involves the parameter $a$, which implies that our conclusion regarding $\lambda$ is valid for any $a$.

As our second step, we will now use A.23) to simplify our original polynomial (3.21). To be explicit, (A.23) implies that, in terms of the new coordinates

$$
\begin{aligned}
w & :=\hat{h}^{0}+2 \lambda \hat{h}^{4} \\
v & :=\hat{h}^{0}-\lambda \hat{h}^{4},
\end{aligned}
$$

the polynomial (3.21) simplifies to

$$
\hat{\mathcal{V}}\left(w, v, \hat{h}^{A}\right)=w v^{2}-\frac{1}{2}\left[w\left(1+\frac{\kappa}{\lambda}\right)+v\left(2-\frac{\kappa}{\lambda}\right)\right] \delta_{A B} \hat{h}^{A} \hat{h}^{B} .
$$

We will now forget about the original form (3.21), as well as the corresponding embedding equations (A.11)-(A.20). Instead, we will now exclusively use the new form (A.27), because the corresponding embedding equations are much easier to solve.

That is, we now parameterize the embedding as

$$
\begin{aligned}
w & =A X+B Y+C Z \\
\hat{h}^{1} & =D Y \\
\hat{h}^{2} & =E Y \\
\hat{h}^{3} & =F Y \\
v & =G X+H Y+I Z
\end{aligned}
$$


with $\left(D^{2}+E^{2}+F^{2}\right) \neq 0$. Note that the coefficients here have nothing to do with the old coefficients $A, \ldots, I$ we used earlier.

Inserting now this ansatz into (A.27) and comparing with (A.5) yields the new embedding conditions

$$
\begin{aligned}
A G^{2} & =\frac{1}{3} \\
B H^{2}-\frac{1}{2} R^{2}\left[B\left(1+\frac{\kappa}{\lambda}\right)+H\left(2-\frac{\kappa}{\lambda}\right)\right] & =a \\
C I^{2} & =0 \\
A H^{2}+2 B G H-\frac{1}{2} R^{2}\left[A\left(1+\frac{\kappa}{\lambda}\right)+G\left(2-\frac{\kappa}{\lambda}\right)\right] & =0 \\
A I^{2}+2 C G I & =0 \\
B G^{2}+2 A G H & =0 \\
C G^{2}+2 A G I & =1 \\
B I^{2}+2 C H I & =0 \\
C H^{2}+2 B H I-\frac{\kappa}{2} R^{2}\left[C\left(1+\frac{\kappa}{\lambda}\right)+I\left(2-\frac{\kappa}{\lambda}\right)\right] & =0 \\
2[A H I+B G I+C G H] & =1,
\end{aligned}
$$

where again $R^{2} \equiv D^{2}+E^{2}+F^{2}$. We will now solve these equations. Our strategy is to first solve $A, B, C, H, I$ in terms of $G$ and then relate $G$ to the other parameters. We start from eq. (A.33), which implies $A, G \neq 0$ and

$$
A=\frac{1}{3 G^{2}}
$$

$C$ can be either zero or not. If $C=0$, A.37) implies (because of $A \neq 0$ ) that $I=0$. However, (A.42) forbids that $C$ and $I$ vanish simultaneously, so that we have to assume $C \neq 0$. But then, (A.35) tells us that

$$
I=0 .
$$

This then automatically solves (A.37) and (A.40). From (A.39) we obtain

$$
C=\frac{1}{G^{2}}
$$

(A.42) then implies

$$
H=\frac{G}{2} .
$$

From eq. (A.38), we then obtain

$$
B=-\frac{1}{3 G^{2}}
$$

Eq. (A.41) implies

$$
R^{2}\left(1+\frac{\kappa}{\lambda}\right)=2 H^{2}
$$


which gives us a constraint on $\kappa$ :

$$
\kappa>-\lambda
$$

From eqn. (A.36), we learn that

$$
R^{2}\left(2-\frac{\kappa}{\lambda}\right)=-\frac{2}{3 G}
$$

which gives us another constraint on $\kappa$ :

$$
\kappa \neq 2 \lambda
$$

as well as the consistency conditions (because of $R^{2}>0$ )

$$
\begin{aligned}
G>0 & \Leftrightarrow \kappa>2 \lambda \\
G<0 & \Leftrightarrow \kappa<2 \lambda .
\end{aligned}
$$

Adding (A.48) and (A.50) gives $R^{2}$ in terms of $G$ :

$$
R^{2}=\frac{G^{2}}{6}-\frac{2}{9 G}
$$

whereas dividing (A.48) by A.50) (which is always possible because of $R^{2} \neq 0$ and (A.51) yields $G$ in terms of $\kappa$ :

$$
G^{3}=-\frac{4(\lambda+\kappa)}{3(2 \lambda-\kappa)}
$$

One now convinces oneself that any value for $\kappa$ in the allowed region (cf. eqs. (A.49), (A.51)

$$
\kappa \in(-\lambda, 2 \lambda) \cup(2 \lambda,+\infty)
$$

gives a value for $G$ via (A.55) that automatically also satisfies the consistency conditions (A.52)-(A.53) and gives rise to a positive value for $R^{2}$ via (A.54). The other coefficients $A, B, C, H, I$ can then be unambiguously calculated from $G$ using the above equations.

At this point, we have therefore shown that any $\kappa \in(-\lambda, 2 \lambda) \cup(2 \lambda,+\infty)$ leads to a consistent solution to eq. (A.33) and eqs. (A.35)-( A.42). The only equation we have not yet used/solved, is equation (A.34), which is the only equation involving the parameter $a$. Thus, all we have derived so far applies to all possible $a$. Inserting our results into eq. (A.34), however, fixes $a$ to be

$$
a=\frac{1}{6}
$$

without that any new constraints are imposed on the other coefficients. Remarkably, $a=\frac{1}{6}$ exactly corresponds to the value that is favored if one takes the one-loop threshold effects into account (see Section 3.3). 
Putting everything together, the embedding of (A.5) into (3.21) fixes $\lambda$ and $a$, and restricts $\kappa$ to take on values in two intervals:

$$
\begin{aligned}
& a=\frac{1}{6} \\
& \lambda=\frac{1}{\sqrt{2}} \\
& \kappa \in\left(-\frac{1}{\sqrt{2}}, \sqrt{2}\right) \cup(\sqrt{2},+\infty) .
\end{aligned}
$$

\section{B. All the allowed $\kappa$ lead to equivalent $\hat{\mathcal{M}}$}

Naively, one might now conclude that the extended scalar manifold $\hat{\mathcal{M}}$ is not unique, and that, instead, there is a one-parameter family of such manifolds parameterized by the allowed values for $\kappa$. We will now show that this is not true, because all the allowed $\kappa$ lead to equivalent manifolds.

To be precise, we will show that any $\kappa_{1} \in(-\lambda, 2 \lambda) \cup(2 \lambda,+\infty)$ can be transformed into any other $\kappa_{2} \in(-\lambda, 2 \lambda) \cup(2 \lambda,+\infty)$ by means of a simple rescaling of the variables $\left(v, w, \hat{h}^{A}\right)$ of the polynomial (A.27)

To see this, choose two arbitrary allowed $\kappa$ values $\kappa_{1}, \kappa_{2} \in(-\lambda, 2 \lambda) \cup(2 \lambda,+\infty)$.

Then define

$$
\begin{aligned}
\rho & :=\left(\frac{\lambda+\kappa_{1}}{\lambda+\kappa_{2}}\right)^{\frac{1}{6}}\left(\frac{2 \lambda-\kappa_{1}}{2 \lambda-\kappa_{2}}\right)^{\frac{1}{3}} \\
\sigma & :=\left(\frac{2 \lambda-\kappa_{2}}{2 \lambda-\kappa_{1}}\right) \rho^{2} .
\end{aligned}
$$

Note that $\left(\lambda+\kappa_{i}\right)>0$ and $\kappa_{i} \neq 2 \lambda$, so that $\rho$ and $\sigma$ are well-defined, non-vanishing real numbers. Now consider the polynomial (A.27) with $\kappa=\kappa_{1}$,

$$
\hat{\mathcal{V}}\left(w, v, \hat{h}^{A}\right)=w v^{2}-\frac{1}{2}\left[w\left(1+\frac{\kappa_{1}}{\lambda}\right)+v\left(2-\frac{\kappa_{1}}{\lambda}\right)\right] \delta_{A B} \hat{h}^{A} \hat{h}^{B} .
$$

After the coordinate rescalings

$$
\begin{aligned}
v & \longrightarrow \sigma v \\
w & \longrightarrow \sigma^{-2} w \\
\hat{h}^{A} & \longrightarrow \rho^{-1} \hat{h}^{A}
\end{aligned}
$$

this becomes

$$
\hat{\mathcal{V}}\left(w, v, \hat{h}^{A}\right)=w v^{2}-\frac{1}{2}\left[w\left(1+\frac{\kappa_{2}}{\lambda}\right)+v\left(2-\frac{\kappa_{2}}{\lambda}\right)\right] \delta_{A B} \hat{h}^{A} \hat{h}^{B},
$$

which is nothing but (A.27) with $\kappa=\kappa_{2}$. Thus all our polynomials A.27) with $\kappa \in(-\lambda, 2 \lambda) \cup$ $(2 \lambda,+\infty)$ are equivalent and describe the same scalar manifold $\hat{\mathcal{M}}$, which is thus unique. 


\section{References}

[1] E. Witten, Phases of $N=2$ Theories in Two Dimensions, Nucl. Phys. B403 (1993) 159, hep-th/9301042.

P.S. Aspinwall, B.R. Greene and D.R. Morrison, Calabi-Yau Moduli Space, Mirror Manifolds and Spacetime Topology Change in String Theory, Nucl. Phys. B416 (1994) 414, hep-th/9309097.

[2] A. Strominger, Massless Black Holes and Conifolds in String Theory, Nucl. Phys. B451 (1995) 96, hep-th/9504090.

[3] B.R. Greene, D.R. Morrison and A. Strominger, Black Hole Condensation and the Unification of String Vacua, Nucl. Phys. B451 (1995) 109, hep-th/9504145.

[4] A. Klemm and P. Mayr, Strong Coupling Singularities and Non-Abelian Gauge Symmetries in $N=2$ String Theory, Nucl. Phys. B469 (1996) 37, hep-th/9601014.

P. Berglund, S. Katz, A. Klemm and P. Mayr, New Higgs Transitions between Dual $N=2$ String Models, Nucl. Phys. B483 (1997) 209, hep-th/9605154.

[5] S. Katz, D. R. Morrison, M. R. Plesser, Enhanced Gauge Symmetry in Type II String Theory, Nucl. Phys. B477 (1996) 105, hep-th/9601108.

[6] D.R. Morrison and C. Vafa, Compactifications of F-Theory on Calabi-Yau Threefolds - I, Nucl. Phys. B473 (1996) 74, hep-th/9602114.

D.R. Morrison and C. Vafa, Compactifications of F-Theory on Calabi-Yau Threefolds - II, Nucl. Phys. B476 (1996) 437, hep-th/9603161.

[7] E. Witten, Phase Transitions in M-Theory and F-Theory, Nucl. Phys. B471 (1996) 195, hep-th/9603150.

[8] D.R. Morrison and N. Seiberg, Extremal Transitions and Five-Dimensional Supersymmetric Field Theories, Nucl. Phys. B483 (1997) 229, hep-th/9609070.

K. Intrilligator, D.R. Morrison and N. Seiberg, Five-Dimensional Supersymmetric Gauge Theories and Degenerations of Calabi-Yau Spaces, Nucl. Phys. B497 (1997) 56, hep-th/9702198.

[9] S. Kachru, A. Klemm, W. Lerche, P. Mayr and C. Vafa, Nonperturbative Results on the Point Particle Limit of $N=2$ Heterotic String Compactifications, Nucl. Phys. B459 (1996) 537, hep-th/9508155.

[10] S.B. Giddings, S. Kachru and J. Polchinski, Hierarchies from Fluxes in String Compactifications, hep-th/0105097.

[11] I.R. Klebanov and M.J. Strassler, Supergravity and a confining gauge theory: duality cascades and $\chi$ sb-resolution of naked singularities, JHEP 0008 (2000) 052, hep-th/0007191.

[12] J.L. Feng, J. March-Russel, S. Sethi and F. Wiczek, Saltatory Relaxation of the Cosmological Constant, Nucl. Phys. B602 (2001) 307, hep-th/0005276.

[13] M. Dine, TASI Lectures on M Theory Phenomenology, hep-th/0003175.

[14] M. Günaydin, G. Sierra and P.K. Townsend, The geometry of $N=2$ Maxwell-Einstein supergravity and Jordan algebras, Nucl. Phys. B242 (1984) 244

[15] M. Günaydin, G. Sierra and P.K. Townsend, Gauging the $d=5$ Maxwell/Einstein supergravity theories: More on Jordan algebras Nucl. Phys. B253 (1985), 573 
[16] M. Günaydin and M. Zagermann, The gauging of five-dimensional, $\mathcal{N}=2$ Maxwell-Einstein supergravity theories coupled to tensor multiplets, Nucl. Phys. B572 (2000) 131-150, hep-th/9912027

[17] E. Cremmer, B. Julia and J. Scherk, Supergravity Theory in Eleven Dimenisons, Phys. Lett. B76 (1978) 409.

[18] A.C. Cadavid, A. Ceresole, R. D'Auria, S. Ferrara, 11-Dimensional Supergravity Compactified on Calabi-Yau Threefolds, Phys.Lett. B357 (1995) 76, hep-th/9506144;

G. Papadopoulos, P.K. Townsend, Compactification of D=11 supergravity on spaces of exceptional holonomy, Phys.Lett. B357 (1995) 300, hep-th/9506150.

[19] I. Antoniadis, S. Ferrara, T.R. Taylor, N=2 Heterotic Superstring and its Dual Theory in Five Dimensions, Nucl.Phys. B460 (1996) 489, hep-th/9511108.

[20] A. Giveon, M. Porrati and E. Rabinovici, Target Space Duality in String Theory, Phys. Rept. 244 (1994) 77, hep-th/9401139.

[21] L. Ibanez, W. Lerche, D. Lüst and S. Theisen, Some Considerations about the Stringy Higgs Effect, Nucl. Phys. B352 (1991) 435.

[22] G. Sierra, $N=2$ Maxwell matter Einstein supergravities in $d=5, d=4$ and $d=3$, Phys. Lett. B157 (1985) 379.

[23] B. de Wit and A. van Proeyen, Special geometry, cubic polynomials and homogeneous quaternionic spaces, Commun. Math. Phys. 149 (1992) 307; hep-th/9112027

[24] J. Louis, J. Sonnenschein, S. Theisen and S. Yankielowicz, Non-Perturbative Properties of Heterotic String Vacua Compactified on K3 $\times T^{2}$, Nucl.Phys. B480 (1996) 185-212, hep-th/9606049.

[25] A. Ceresole and G. Dall'Agata, General matter coupled N=2, D=5 gauged supergravity, Nucl.Phys. B585 (2000) 143-170, hep-th/0004111.

[26] J. Ellis, M. Günaydin and M. Zagermann, Options for Gauge Groups in Five-Dimensional Supergravity, hep-th/0108094

[27] B. de Wit and A. van Proeyen, Broken sigma-model isometries in very special geometry, Phys. Lett. B293 (1992) 94; hep-th/9207091

[28] B. de Wit and A. Van Proeyen, Potentials and Symmetries of General Gauged $N=2$ Supergravity - Yang-Mills Models, Nucl. Phys. B245 (1984) 89.

[29] L. Andrianopoli, M. Bertolini, A. Cresole, R. D'Auria,S. Ferrara, P. Fré and T. Magri, $N=2$ Supergravity and $N=2$ Super Yang-Mills Theory on General Scalar Manifolds, J. Geom. Phys. 23 (1997) 111, hep-th/9605032.

[30] A. Chou, R. Kallosh, J. Rahmfeld, S.-J. Rey, M. Shmakova and W.K. Wong, Critical Points and Phase Transitions in 5D Compactifications of M-Theory, Nucl. Phys. B508 (1997) 147, hep-th/9704142.

[31] M. Günaydin, G. Sierra and P.K. Townsend, More on $d=5$ Maxwell-Einstein supergravity: symmetric spaces and kinks, Class. Quantum Grav. 3 (1986) 763 
[32] B. de Wit, V. Kaplunovsky, J. Louis and D. Lüst, Perturbative Couplings of Vector Multiplets in $N=2$ Heterotic String Vacua, Nucl. Phys. B451 (1995) 53, hep-th/9504006.

I. Antoniadis, S. Ferrara, E. Gava, K.S. Narain and T.R. Taylor, Perturbative Prepotentials and Monodromies in $N=2$ Heterotic Superstring, Nucl. Phys. B447 (1995) 35, hep-th/9504034.

[33] T. Mohaupt, in preparation.

[34] R. Kallosh, T. Mohaupt and M. Shmakova, Excision of Singularities by Stringy Domain Walls, Jour. Math. Phys. 42 (2001) 3071, hep-th/0010271.

[35] I. Gaida, S. Mahapatra, T. Mohaupt and W.A. Sabra, Black Holes and Flop Transitions in M-Theory on Calabi-Yau Threefolds, Class. Quant. Grav. 16 (1999) 419, hep-th/9807014.

[36] B.R. Greene, K. Schalm and G. Shiu, Dynamical Topology Change in M Theory, hep-th/0010207.

[37] L. Randall and R. Sundrum, "An alternative to compactification", Phys. Rev. Lett. 83 (1999) 4690, hep-th/9906064; "A large mass hierarchy from a small extra dimension", Phys. Rev. Lett. 83 (1999) 3370, hep-ph/9905221.

[38] R. Altendorfer, J. Bagger and D. Nemeschansky, "Supersymmetric Randall-Sundrum scenario", hep-th/0003117; T. Gherghetta and A. Pomarol, "Bulk fields and supersymmetry in a slice of AdS", Nucl. Phys. B 586 (2000) 141, hep-ph/0003129; A. Falkowski, Z. Lalak and S. Pokorski, "Supersymmetrizing branes with bulk in five-dimensional supergravity", Phys. Lett. B 491 (2000) 172, hep-th/0004093; A. Falkowski, Z. Lalak and S. Pokorski, "Five-dimensional gauged supergravities with universal hypermultiplet and warped brane worlds", hep-th/0009167;

E. Bergshoeff, R. Kallosh and A. Van Proeyen, "Supersymmetry in singular spaces", JHEP0010 (2000) 033, hep-th/0007044; M. J. Duff, J. T. Liu and K. S. Stelle, "A supersymmetric type IIB Randall-Sundrum realization", hep-th/000712d.

[39] K. Behrndt, S. Gukov, Domain Walls and Superpotentials from M Theory on Calabi-Yau Three-Folds, Nucl.Phys. B580 (2000) 225-242, hep-th/0001082.

[40] A. Lukas, B. A. Ovrut, K. S. Stelle and D. Waldram, The universe as a domain wall, Phys. Rev. D59 (1999) 086001, hep-th/9803235, Heterotic M theory in Five Dimensions, Nucl.Phys. B552 (1999) 246, hep-th/9806051.

[41] M. Haack, Ph.D. thesis, 2001, Halle University, to appear in Fortschr. Phys., currently available at http://www .physik.uni-halle.de/Fachgruppen/Theorie/qft/arbeiten/arbeiten.html.

[42] C. V. Johnson, A. W. Peet, J. Polchinski, Gauge Theory and the Excision of Repulson Singularities, Phys.Rev. D61 (2000) 086001, hep-th/9911161.

[43] P. Hořava and E. Witten, Heterotic and type I string dynamics from eleven dimensions, Nucl.Phys. B460 (1996) 506, hep-th/9510209;

Eleven-Dimensional Supergravity on a Manifold with Boundary, Nucl. Phys. B475 (1996) 94, hep-th/9603142;

P. Hořava, Gluino condensation in strongly coupled heterotic string theory, Phys. Rev. D54 (1996) 7561, hep-th/9608019.

[44] J. Khoury, B. A. Ovrut, N. Seiberg, P. J. Steinhardt, N. Turok, From Big Crunch to Big Bang, hep-th/0108187. 
[45] B. Szendröi, Diffeomorphisms and families of Fourier-Mukai transforms in mirror symmetry, math.AG/0103137. 